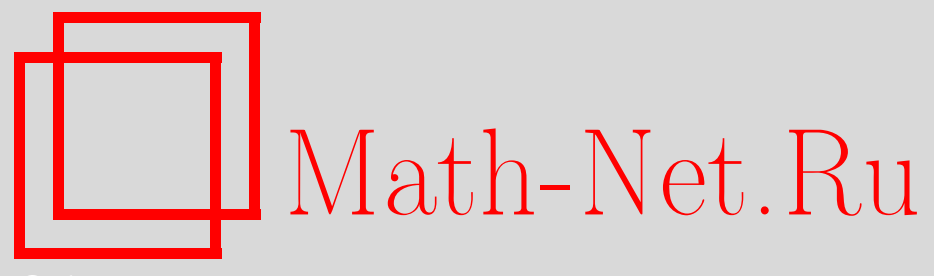

А. А. Белавин, Ал. Б. Замолодчиков, Интегралы по пространству модулей, кольцо дискретных состояний и четырехточечная функция в минимальной лиувиллевской гравитации, ТМФ, 2006, том 147, номер 3, 339-371

DOI: https://doi.org/10.4213/tmf1984

Использование Общероссийского математического портала Math-Net.Ru подразумевает, что вы прочитали и согласны с пользовательским соглашением http://www . mathnet.ru/rus/agreement

Параметры загрузки:

IP : 54.84 .234 .179

26 апреля 2023 г., 14:15:48

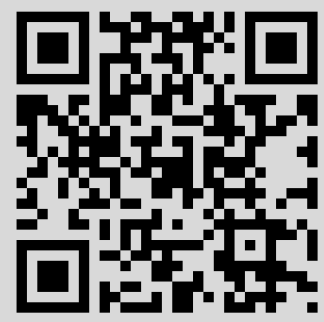




\title{
ИНТЕГРАЛЫ ПО ПРОСТРАНСТВУ МОДУЛЕЙ, КОЛЬЦО ДИСКРЕТНЫХ СОСТОЯНИЙ И ЧЕТЫРЕХТОЧЕЧНАЯ ФУНКЦИЯ В МИНИМАЛЬНОЙ ЛИУВИЛЛЕВСКОЙ ГРАВИТАЦИИ
}

\begin{abstract}
Прямое вычисление корреляционных функций в двумерной минимальной гравитации связано с интегрированием по пространству модулей. Для вырожденных полей высшие уравнения движения теории поля Лиувилля позволяют превратить подынтегральное выражение в производную, при этом весь интеграл сводится к граничным вкладам и к так называемому вкладу от кривизны. Последний напрямую связан с вакуумным ожиданием от соответствующего элемента кольца дискретных состояний. Действие этого элемента на когомологии, связанные с примарными полями материи общего вида, может быть непосредственно вычислено с помощью разложения операторных произведений вырожденных полей. На основании этого построена алгебра на кольце дискретных состояний и вычислен вклад кривизны в четырехточечную функцию. Проанализированы разложения операторных произведений для лиувиллевских "логарифмических примарных полей” и вычислены существенные логарифмические члены. На основании этого получено явное выражение для четырехточечного корреляционного числа одного вырожденного и трех общих полей материи. Этот интеграл сравнивается с числами, полученными с использованием матричных моделей двумерной гравитации, и обсуждаются некоторые связанные с этим задачи и неоднозначности.
\end{abstract}

Ключевые слова: поляковская теория струн, лиувиллевская гравитация.

\section{1. ВВЕДЕНИЕ}

1. Лиувиллевская гравитация (ЛГ) обозначает двумерную квантовую гравитацию с действием, индуцированным "критической" материей, т.е. материей $\mathcal{M}_{c}$,

* Институт теоретической физики им. Л. Д. Ландау РАН, Черноголовка, Московская обл., Россия. E-mail: belavin@itp.ac.ru

${ }^{\dagger}$ Laboratoire de Physique Théorique et Astroparticules Université Montpellier II, Montpellier, France; Институт теоретической и экспериментальной физики, Москва, Россия. E-mail: Aliocha.ZAMOLODCHIKOV@lpta.univ-montp2.fr 
описываемой конформной теорией поля (КТП) с центральным зарядом $c$. Это индуцированное действие оказывается универсальным и носит название действия Лиувилля, поскольку его вариация по отношению к метрике пропорциональна уравнению Лиувилля (или уравнению постоянной кривизны) [1]. Обозначим через $\left\{\Phi_{i}, \Delta_{i}\right\}$ набор примарных полей и их размерностей в КТП материи $\mathcal{M}_{c}$.

2. Теория поля Лиувилля (ТПЛ) строится как квантовая версия классической теории, основанная на действии Лиувилля. ТПЛ снова представляет собой КТП с центральным зарядом $c_{\mathrm{L}}$. Его удобно параметризовать в терминах параметра $b$ или параметра

$$
Q=b^{-1}+b
$$

KaK

$$
c_{\mathrm{L}}=1+6 Q^{2} .
$$

Параметр $b$ входит в локальный лагранжиан

$$
\mathcal{L}_{\mathrm{L}}=\frac{1}{4 \pi}\left(\partial_{a} \phi\right)^{2}+\mu e^{2 b \phi}
$$

где $\mu$ - масштабный параметр, называемый космологической постоянной, а $\phi-$ динамическая переменная для квантованной метрики

$$
d s^{2}=e^{2 b \phi} \hat{g}_{a b} d x^{a} d x^{b} .
$$

В этом выражении $\hat{g}_{a b}$ представляет собой "фоновую метрику” - технический прием, необходимый для приведения ТПЛ к ковариантному виду:

$$
\mathcal{A}_{\mathrm{L}}=\int\left(\frac{1}{4 \pi} \hat{g}^{a b} \partial_{a} \phi \partial_{b} \phi+\frac{Q}{4 \pi} \phi \widehat{R}+\mu e^{2 b \phi}\right) \sqrt{\hat{g}} d^{2} x,
$$

где через $\widehat{R}$ обозначена скалярная кривизна фоновой метрики. Базисные примарные поля задаются экспоненциальными операторами $V_{a}=e^{2 a \phi}$, параметризованными непрерывным (в общем случае комплексным) параметром $a$ таким образом, что соответствующая конформная размерность имеет вид

$$
\Delta_{a}^{(\mathrm{L})}=a(Q-a)
$$

ТПЛ решается точно [2]. В частности, трехточечная функция $C_{\mathrm{L}}\left(a_{1}, a_{2}, a_{3}\right)=$ $\left\langle V_{a_{1}}\left(x_{1}\right) V_{a_{2}}\left(x_{2}\right) V_{a_{3}}\left(x_{3}\right)\right\rangle_{\mathrm{L}}$ известна в явном виде для произвольных экпоненциальных полей,

$$
C_{\mathrm{L}}\left(a_{1}, a_{2}, a_{3}\right)=\left(\pi \mu \gamma\left(b^{2}\right) b^{2-2 b^{2}}\right)^{(Q-a) / b} \frac{\Upsilon_{b}(b)}{\Upsilon_{b}(a-Q)} \prod_{i=1}^{3} \frac{\Upsilon_{b}\left(2 a_{i}\right)}{\Upsilon_{b}\left(a-a_{i}\right)},
$$

где $a=a_{1}+a_{2}+a_{3}$, а символ $\Upsilon_{b}(x)$ обозначает специальную функцию, связанную с двойной гамма-функцией Барнса (см., например, [3]). Корреляционная функция (1.7) оказывается согласованной со следующим отождествлением экспоненциальных полей при различных значениях параметра $a$ :

$$
V_{a}(x)=R_{\mathrm{L}}(a) V_{Q-a}(x),
$$


известным как соотношения отражения [3]. Здесь

$$
R_{\mathrm{L}}(a)=\left(\pi \mu \gamma\left(b^{2}\right)\right)^{(Q-2 a) / b} \frac{\gamma\left(2 a b-b^{2}\right)}{\gamma\left(2-2 a b^{-1}+b^{-2}\right)}
$$

- так называемая лиувиллевская амплитуда отражения. Локальная структура ТПЛ полностью определяется общим "непрерывным" операторным разложением (ОР) для экспоненциальных полей Лиувилля общего вида:

$$
V_{a_{1}}(x) V_{a_{2}}(0)=\int^{\prime} \frac{d P}{4 \pi} C_{a_{1}, a_{2}}^{(\mathrm{L}) Q / 2+i P}(x \bar{x})^{\Delta_{Q / 2+i P}^{(\mathrm{L})}-\Delta_{a_{1}}^{(\mathrm{L})}-\Delta_{a_{2}}^{(\mathrm{L})}}\left[V_{Q / 2+i P}(0)\right],
$$

где структурная константа выражена через функцию (1.7): $C_{a_{1}, a_{2}}^{(\mathrm{L}) p}=C_{\mathrm{L}}(g, a, Q-p)$. Контур интегрирования в этом выражении есть ось вещественных чисел, если параметры $a_{1}$ и $a_{2}$ лежат в "главной области"

$$
\left|\frac{Q}{2}-\operatorname{Re} a_{1}\right|+\left|\frac{Q}{2}-\operatorname{Re} a_{2}\right|<\frac{Q}{2} .
$$

При других значениях этих параметров необходимо производить аналитическое продолжение, которое эквивалентно определенным деформациям контура, возникающим из-за сингулярностей структурной константы. Штрих возле символа интегрирования в (1.10) обозначает это предписание.

Параметр $b$ в ЛГ выбирается таким образом, чтобы вместе с КТП материи $\mathcal{M}_{c}$ ТПЛ образовывала бы совокупную КТП с центральным зарядом $c+c_{\mathrm{L}}=26$. Также из технических соображений удобно рассмотреть следующую теорию.

3. Теория поля репараметризационных духов представляет собой стандартную фермионную $B C$-систему со спином $(2,-1)$ :

$$
A_{\mathrm{gh}}=\frac{1}{\pi} \int(C \bar{\partial} B+\bar{C} \partial \bar{B}) d^{2} x
$$

с центральным зарядом - 26, который соответствует детерминанту Фаддеева-Попова, фиксирующему калибровку. Тензор напряженности $T$ для системы "материя+поле Лиувилля" представляет собой генератор 26-мерной алгебры Вирасоро. В совокупности с духовой полевой теорией это позволяет построить БРСТ-комплекс с нильпотентным БРСТ-зарядом вида

$$
\mathcal{Q}=\oint(C T+C \partial C B) \frac{d z}{2 \pi i} .
$$

4. Корреляционные функции. Построение корреляционных функций представляет собой одну из наиболее важных задач в ЛГ. В гравитационных корреляционных функциях операторы материи $\Phi_{i}$ "одеваются" соответствующими экспоненциальными полями Лиувилля $V_{a_{i}}$ таким образом, что они становятся либо $(1,1)$ формами $U_{i}=\Phi_{i} V_{a_{i}}$ с нулевым духовым числом, либо операторами $W_{i}=C \bar{C} U_{i}$ размерности $(0,0)$ с духовым числом 1 . В обоих случаях должно выполняться условие

$$
\Delta_{i}+a_{i}\left(Q-a_{i}\right)=1 .
$$


Инвариантные (или проинтегрированные) корреляционные функции не зависят от координат, и потому правильнее их называть корреляционными числами. В теоретико-полевом подходе (для рода ноль) корреляционное число $\left\langle U_{1} \ldots U_{n}\right\rangle_{\mathrm{G}}$ при $n \geqslant 3$ представляется интегралом

$$
\left\langle U_{1} \ldots U_{n}\right\rangle_{\mathrm{G}}=\int\left\langle W_{1}\left(x_{1}\right) W_{2}\left(x_{2}\right) W_{3}\left(x_{3}\right) U_{4}\left(x_{4}\right) d^{2} x_{4} \ldots U_{n}\left(x_{n}\right) d^{2} x_{n}\right\rangle .
$$

Здесь интегрирование производится по пространству модулей $M_{n}$ сферы с $n$ отмеченными точками. Технически это эквивалентно выбору любых трех вставок $W_{i}$ в произвольных фиксированных точках $x_{1}, x_{2}$ и $x_{3}$ с последующим интегрированием $(1,1)$-форм $U_{i}\left(x_{i}\right) d^{2} x_{i}$, вставленных вместо $W_{i}$ в точках $i=4, \ldots, n$. Это определение отчасти модифицируется при $n<3$ ввиду наличия нетривиальных конформных симметрий сферы с двумя выколотыми точками и без выколотых точек.

Простейший случай корреляционной функции (1.15) представляет собой трехточечную функцию; при этом пространство модулей тривиально, а результат сводится к произведению трехточечных функций полей материи, поля Лиувилля и полей духов:

$$
\left\langle U_{1} U_{2} U_{3}\right\rangle_{\mathrm{G}}=x_{12} \bar{x}_{12} x_{23} \bar{x}_{23} x_{31} \bar{x}_{31}\left\langle\Phi_{1}\left(x_{1}\right) \Phi_{2}\left(x_{2}\right) \Phi_{3}\left(x_{3}\right)\right\rangle_{\mathrm{CFT}}\left\langle V_{1}\left(x_{1}\right) V_{2}\left(x_{2}\right) V\left(x_{3}\right)\right\rangle_{\mathrm{L}}
$$

Трехточечные функции $\left\langle\Phi_{1}\left(x_{1}\right) \Phi_{2}\left(x_{2}\right) \Phi_{3}\left(x_{3}\right)\right\rangle_{\text {СғT }}$, также известные как структурные константы алгебры ОР, были явно построены в решаемых КТП с материей. Таким образом, уравнение (1.16) задает в явном виде трехточечное корреляционное число ЛГ. Двухточечное число и нуль-точечное число (статистическая сумма) могут быть легко получены из данного выражения для трехточечной функции.

5. Четырехточечная функция представляет собой следующий этап по уровню сложности:

$$
\left\langle U_{1} U_{2} U_{3} U_{4}\right\rangle_{\mathrm{G}}=x_{12} \bar{x}_{12} x_{23} \bar{x}_{23} x_{31} \bar{x}_{31} \int\left\langle\Phi_{1}\left(x_{1}\right) \ldots \Phi_{4}\left(x_{4}\right)\right\rangle_{\mathrm{CFT}}\left\langle V_{1}\left(x_{1}\right) \ldots V_{4}\left(x_{4}\right)\right\rangle_{\mathrm{L}} d^{2} x_{4} .
$$

Это выражение намного менее явно. Во-первых, оно содержит интегрирование по $x_{4}$. Далее, даже если четырехточечная функция материи известна в сколь-либо подходящем виде, общий вид представления для лиувиллевской четырехточечной функции оказывается намного более сложным. Например, разложение "конформных блоков" [3]

$$
\left\langle V_{1}\left(x_{1}\right) \ldots V_{4}\left(x_{4}\right)\right\rangle_{\mathrm{L}}=\int \frac{d P}{4 \pi} C_{\mathrm{L}}\left(a_{1}, a_{2}, \frac{Q}{2}+i P\right) C_{\mathrm{L}}\left(\frac{Q}{2}-i P, a_{3}, a_{4}\right) \mathcal{F}_{P}\left(a_{i}, x_{i}\right) \mathcal{F}_{P}\left(a_{i}, \bar{x}_{i}\right)
$$

содержит так называемый общий конформный блок [4]

$$
\mathcal{F}_{P}\left(a_{i}, x_{i}\right){ }_{2}^{1} \sum_{4}^{3}=\mathcal{F}_{P}\left(\begin{array}{ll|ll}
a_{1} & a_{3} & x_{1} & x_{3} \\
a_{2} & a_{4} & x_{2} & x_{4}
\end{array}\right),
$$


который сам представляет собой сложную функцию своих аргументов, не говоря уже об интегрировании по “промежуточному импульсу" $P$ в формуле (1.18). В данной работе мы делаем предварительный шаг по направлению к вычислению четырехточечного интеграла в специальном случае теории с минимальной гравитацией.

6. Минимальная гравитация. Если конформная материя $\mathcal{M}_{c}$ задается минимальной моделью КТП (более точно, “обобщенной минимальной моделью" (OMM), см. ниже) $\mathcal{M}_{b^{2}}$, то можно говорить про "минимальную гравитацию" (МГ) (соответственно про обобщенную минимальную гравитацию (ОМГ)). В ОМГ вычисление четырехточечного интеграла существенно упрощается, если один из операторов материи $\Phi_{i}$ в правой части (1.17) оказывается вырожденным полем $\Phi_{m, n}$. Это происходит благодаря так называемым "высшим уравнениям движения" (ВУД), выполненным для операторных полей в ТПЛ [5]. Пусть $U_{4}=U_{m, n}$, где

$$
U_{m, n}=\Phi_{m, n} \widetilde{V}_{m, n},
$$

а $\widetilde{V}_{m, n}$ - подходящее лиувиллевское одевание для $\Phi_{m, n}$. Тогда с помощью ВУД можно переписать подынтегральное выражение в (1.17) в виде производной,

$$
\left\langle U_{1} U_{2} U_{3} U_{m, n}\right\rangle_{\mathrm{OM} \Gamma}=B_{m, n}^{-1} \int \partial \bar{\partial}\left\langle O_{m, n}^{\prime}(x) W_{1}\left(x_{1}\right) W_{2}\left(x_{2}\right) W_{3}\left(x_{3}\right)\right\rangle d^{2} x
$$

(здесь $B_{m, n}$ - числовая постоянная, см. раздел 3 ), и тем самым свести задачу к граничным членам и к так называемому вкладу от кривизны. Последний непосредственно выражается с помощью вакуумного среднего $\left\langle O_{m, n} W_{1} W_{2} W_{3}\right\rangle$ элемента $O_{m, n}$ кольца дискретных состояний.

7. Кольцо дискретных состояний (КДС). Элемент КДС $O_{m, n}$ связан с полем $\Phi_{m, n}$. Мы намерены исследовать, как обращаться с алгеброй КДС и с корреляционными функциями ее элементов. Это знание окажется полезным и при последующих вычислениях граничных членов.

8. Граничные члены. Для того чтобы вычислить граничные вклады, необходимо знать соответствующие слагаемые в ОР полей $O_{m, n}^{\prime}$ в формуле (1.21). Это поле строится как "логарифмический аналог" элемента $O_{m, n}$ и удовлетворяет тождеству

$$
\partial \bar{\partial} O_{m, n}^{\prime}=B_{m, n} U_{m, n}+\text { БРСТ-точные члены. }
$$

Анализируя это разложение, можно вычислить граничные вклады и в конце концов построить четырехточечный интеграл (1.17) с $U_{4}=U_{m, n}$. В этом состоит главная задача представленной ниже работы.

\section{2. ОБОБЩЕННЫЕ МИНИМАЛЬНЫЕ МОДЕЛИ}

Строго говоря, минимальные модели КТП $\mathcal{M}_{p / p^{\prime}}$ [4] определены согласованным образом, только если "параметр" $p / p^{\prime}$ представляет собой несократимое рациональное число, т.е. если целые числа $p$ и $p^{\prime}$ взаимно просты. В этом случае конечный набор из $(p-1)\left(p^{\prime}-1\right) / 2$ вырожденных примарных полей $\Phi_{m, n}$ с $1 \leqslant m<p$ и $1 \leqslant n<p^{\prime}$ (по модулю отождествления $\Phi_{m, n}=\Phi_{p-m, p^{\prime}-n}$ ) образует вместе со своими неприводимыми представлениями полное пространство модели $\mathcal{M}_{p / p^{\prime}}$. Представляется, что 
"канонические" минимальные модели $\mathcal{M}_{p / p^{\prime}}$ являются полностью самосогласованными КТП, т.е. удовлетворяют всем стандартным требованиям квантовой теории поля, кроме (в большинстве случаев) унитарности. Эти модели также считаются точно решаемыми, поскольку структура алгебры их ОР известна в явном виде [6].

Существует много способов снять часть требований, приводящих к набору $\mathcal{M}_{p / p^{\prime}}$ как к единственной возможной структуре КТП. Например, “параметр" $p / p^{\prime}$ часто полагается произвольным числом [6]. При этом алгебра вырожденных примарных полей становится незамкнутой ни на каком конечном подмножестве, и только полный набор $\left\{\Phi_{m, n}\right\}$ с парами $(m, n)$, задаваемыми всеми натуральными числами, образует замкнутую алгебру. Более того, некоторые авторы включают в рассмотрение локальные поля с размерностями, отличными от размерностей Каца, и даже с непрерывным спектром размерностей. Хотя вопрос о согласованности этих конструкций остается открытым с теоретико-полевой точки зрения, эти обобщения доказали свою техническую полезность. Более того, в статистической механике имеется ряд примеров, в которых либо требуется обобщить $\mathcal{M}_{p / p^{\prime}}$ на случай нецелых значений $p / p^{\prime}$, либо невырожденные примарные операторы возникают как наблюдаемые (и оба эти обобщения находят свое применение).

В данной работе $b^{2}$ обозначает параметр $p / p^{\prime}$, а понятие ОММ понимается в наиболее общем смысле КТП с центральным зарядом

$$
c=1-6\left(b^{-1}-b\right)^{2},
$$

в которой могут встречаться поля $\Phi_{\alpha}$ произвольной размерности. Также вводится непрерывный параметр $\alpha$, параметризующий непрерывное семейство примарных полей с размерностями

$$
\Delta_{\alpha}^{(\mathrm{M})}=\alpha(\alpha-q)
$$

где

$$
q=b^{-1}-b .
$$

Также используется “каноническая” нормировка КТП для примарных полей $\Phi_{\alpha}$ через двухточечные функции:

$$
\left\langle\Phi_{\alpha} \Phi_{\alpha}\right\rangle_{\mathrm{OMM}}=(x \bar{x})^{-2 \Delta_{\alpha}} .
$$

Вырожденные поля $\Phi_{m, n}$ имеют размерности

$$
\Delta_{m, n}^{(\mathrm{M})}=-\frac{q^{2}}{4}+\lambda_{m,-n}^{2}
$$

где введено еще одно удобное обозначение

$$
\lambda_{m, n}=\frac{m b^{-1}+n b}{2} .
$$

Эти величины соответствуют либо $\alpha=\alpha_{m, n}$, либо $\alpha=q-\alpha_{m, n}$ при

$$
\alpha_{m, n}=\frac{q}{2}+\lambda_{-m, n}
$$

Главные ограничения, выделяющие указанную достаточно свободную конструкцию, состоят в следующем. 
1. Вырожденные поля $\Phi_{1,2}$ и $\Phi_{2,1}$ (а следовательно, и полный набор $\left\{\Phi_{m, n}\right\}$ в общем случае) лежат в спектре.

2. Нулевые векторы в вырожденных представлениях $\Phi_{m, n}$ обращаются в нуль:

$$
D_{m, n}^{(\mathrm{M})} \Phi_{m, n}=\bar{D}_{m, n}^{(\mathrm{M})} \Phi_{m, n}=0
$$

Здесь $D_{m, n}^{(\mathrm{M})}\left(\bar{D}_{m, n}^{(\mathrm{M})}\right)$ представляют собой операторы, составленные из генераторов правой алгебры Вирасоро $M_{n}{ }^{1)}$ (соответственно из генераторов левой алгебры Вирасоро $\bar{M}_{n}$ ), которые порождают сингулярный вектор на уровне $m n$ для модуля Вирасоро $\Phi_{m, n}$. Для определенности эти операторы нормируются с помощью члена $M_{-1}^{m n}$ как

$$
D_{m, n}^{(\mathrm{M})}=M_{-1}^{m n}+d_{1}^{(m, n)}\left(b^{2}\right) M_{-2} M_{-1}^{m n-2}+\cdots .
$$

Первые примеры могут быть представлены явно:

$$
\begin{aligned}
& D_{1,2}^{(\mathrm{M})}=M_{-1}^{2}-b^{2} M_{-2}, \\
& D_{1,3}^{(\mathrm{M})}=M_{-1}^{3}-2 b^{2}\left(M_{-2} M_{-1}+M_{-1} M_{-2}\right)+4 b^{4} M_{-3},
\end{aligned}
$$

3. Отождествление. Также часто добавляется отождествление $\Phi_{\alpha} \equiv \Phi_{q-\alpha}$.

Оказывается, что этот набор определений накладывает существенные ограничения на структуру указанной формальной конструкции. Например, из вышеприведенных ограничений непосредственно вытекает выражение для трехточечной функции

$$
C_{\mathrm{M}}\left(\alpha_{1}, \alpha_{2}, \alpha_{3}\right)=\left\langle\Phi_{\alpha_{1}} \Phi_{\alpha_{2}} \Phi_{\alpha_{3}}\right\rangle_{\mathrm{OM} \Gamma}
$$

примарных полей "общего вида" [7]:

$$
\begin{aligned}
C_{\mathrm{M}}\left(\alpha_{1}, \alpha_{2}, \alpha_{3}\right)= & \frac{b^{b^{-2}-b^{2}-1} \Upsilon_{b}\left(2 b-b^{-1}+\alpha\right)}{\left[\gamma\left(1-b^{2}\right) \gamma\left(2-b^{-2}\right)\right]^{1 / 2} \Upsilon_{b}(b)} \times \\
& \times \prod_{i=1}^{3} \frac{\Upsilon_{b}\left(\alpha-2 \alpha_{i}+b\right)}{\left[\Upsilon_{b}\left(2 \alpha_{i}+b\right) \Upsilon_{b}\left(2 \alpha_{i}+2 b-b^{-1}\right)\right]^{1 / 2}},
\end{aligned}
$$

где снова $\alpha=\alpha_{1}+\alpha_{2}+\alpha_{3}$ и $\Upsilon_{b}(x)$ - та же функция, что и в равенстве (1.7). При значениях параметров $\alpha_{i}=\alpha_{m_{i}, n_{i}}$ для случая вырождения (при условии, что выполнены стандартные соотношения “слияния"), известные структурные константы [6] можно получить из (2.12).

Явная форма ОР для $\Phi_{1,2}$ и примарного поля общего вида $\Phi_{\alpha}$ имеет вид

$$
\Phi_{1,2}(x) \Phi_{\alpha}(0)=C_{+}^{(\mathrm{M})}(\alpha)(x \bar{x})^{\alpha b}\left[\Phi_{\alpha+b / 2}\right]+C_{-}^{(\mathrm{M})}(\alpha)(x \bar{x})^{1-\alpha b-b^{2}}\left[\Phi_{\alpha-b / 2}\right]
$$

1) Несколько необычное обозначение $M_{n}$ для генераторов Вирасоро конформной симметрии полей материи выбрано для того, чтобы сохранить обозначение $L_{n}$ для генераторов алгебры Вирасоро полей Лиувилля. 
(здесь $\left[\Phi_{\alpha}\right]$ обозначает примарное поле $\Phi_{\alpha}$ вместе со всей башней конформных потомков) и используется ниже. В нашей нормировке

$$
C_{+}^{(\mathrm{M})}(\alpha)=\left[\frac{\gamma\left(b^{2}\right) \gamma\left(2 \alpha b+2 b^{2}-1\right)}{\gamma\left(2 b^{2}-1\right) \gamma\left(b^{2}+2 \alpha b\right)}\right]^{1 / 2} ; \quad C_{-}^{(\mathrm{M})}(\alpha)=\left[\frac{\gamma\left(b^{2}\right) \gamma\left(2 \alpha b+b^{2}-1\right)}{\gamma\left(2 b^{2}-1\right) \gamma(2 \alpha b)}\right]^{1 / 2} .
$$

Общий вид (2.13) записывается следующим образом:

$$
\Phi_{m, n}(x) \Phi_{\alpha}(0)=\sum_{r, s}^{(m, n)}(x \bar{x})^{\lambda_{r, s}\left(2 \alpha+\lambda_{r, s}-q\right)-\Delta_{m, n}^{(\mathrm{M})}} C_{\mathrm{M}}\left(\alpha_{m, n}, \alpha, \alpha+\lambda_{r, s}\right)\left[\Phi_{\alpha+\lambda_{r, s}}\right],
$$

где параметры $\lambda_{r, s}$ те же, что и в формуле (2.6), и символ $\sum_{r, s}^{(m, n)}$ обозначает сумму по следующему набору целых чисел (используется обозначение $\left\{n_{1}: d: n_{1}+n d\right\}=$ $\left.\left\{n_{1}, n_{1}+d, \ldots, n_{1}+n d\right\}\right):$

$$
(r, s)=(\{-m+1: 2: m-1\},\{-n+1: 2: n-1\}) .
$$

Другие точные результаты в ОММ образуют достаточно разноплановую коллекцию. Важным для программы наших исследований является построение четырехточечной функции

$$
G_{(m, n), \alpha_{1}, \alpha_{2}, \alpha_{3}}^{(\mathrm{OMM})}(x)=\left\langle\Phi_{m, n}(x) \Phi_{\alpha_{1}}\left(x_{1}\right) \Phi_{\alpha_{2}}\left(x_{2}\right) \Phi_{\alpha_{3}}\left(x_{3}\right)\right\rangle_{\mathrm{OMM}}
$$

с одним вырожденным полем $\Phi_{m, n}$ и тремя примарными полями общего вида $\Phi_{\alpha}[4]$. Из условия (2.8) отщепления нулевого вектора следует определенное уравнение в частных производных на соответствующие корреляционные функции. В четырехчастичном случае это уравнение сводится к обыкновенному линейному дифференциальному уравнению степени $m n$, независимые решения которого представляют собой четырехточечные конформные блоки

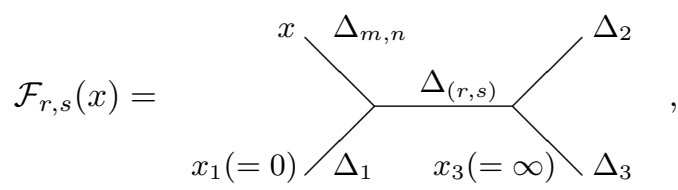

где мы используем обозначения $\Delta_{i}=\Delta_{\alpha_{i}}^{(\mathrm{M})}, \Delta_{m, n}=\Delta_{m, n}^{(\mathrm{M})}$ и $\Delta_{r, s}=\Delta_{\alpha_{1}}^{(\mathrm{M})}+\lambda_{r,-s}\left(2 \alpha_{1}-\right.$ $\left.q+\lambda_{r,-s}\right)$. Четырехточечная функция при этом приобретает вид

$G_{(m, n), \alpha_{1}, \alpha_{2}, \alpha_{3}}^{(\mathrm{OMM})}(x)=\sum_{r, s}^{(m, n)} C_{\mathrm{M}}\left(\alpha_{m, n}, \alpha_{1}, \alpha_{1}+\lambda_{r,-s}\right) C_{\mathrm{M}}\left(\alpha_{1}+\lambda_{r,-s}, \alpha_{2}, \alpha_{3}\right) \mathcal{F}_{r, s}(x) \mathcal{F}_{r, s}(\bar{x})$,

где $\sum_{r, s}^{(m, n)}$ имеет тот же смысл, что и в формуле $(2.15)$.

Следующее замечание весьма важно для развития наших рассуждений. В настоящем исследовании при рассмотрении ОМГ мы ограничиваемся рассмотрением только четырехточечной функции с одним вырожденным полем материи $\Phi_{m, n}$, а оставшиеся три поля считаем формальными полями общего вида $\Phi_{\alpha}$. В частности, 
используя выражение (2.19), можно переписать часть подынтегрального выражения в уравнении (1.17), связанную с полями материи, в удобном виде. Особенно важно то обстоятельство, что если один или более одного из операторов $\Phi_{\alpha}$ также оказываются вырожденными ${ }^{2)}$, то число конформных блоков, входящих в корреляционную функцию (2.19), может уменьшиться, и данное соотношение не будет выполняться буквально. В этом случае нижеприведенные рассуждения также не будут дословно верными. Они требуют существенных и иногда достаточно тонких видоизменений. В данной работе мы не исследуем эту интересную, но более деликатную ситуацию (хотя она оказывается исключительно актуальной в приложениях квантовой гравитации).

Имеется еще одно обстоятельство, схожее с отмеченной выше особенностью теории. При проведении операций в ОММ следует иметь в виду, что мы имеем дело с объектами различной природы. Некоторые из них, например, центральный заряд, размерности вырождения и некоторые корреляционные функции, зависят непрерывно от параметра $b^{2}$. Другие объекты могут вести себя в высшей степени дискретно и зависеть от арифметической природы чисел $p$ и $p^{\prime}$, простейшим примером является число неприводимых представлений алгебры Вирасоро в теории. Из этого ясно, что следует быть аккуратными при воспроизведении результатов для теории $\mathcal{M}_{p / p^{\prime}}$ при взятии предела для $\mathcal{M}_{b^{2}}$ при $b^{2} \rightarrow p / p^{\prime}$ и при $\alpha \rightarrow \alpha_{m, n}$ для формальных примарных полей. Поэтому снова подчеркнем, что три поля материи $\Phi_{\alpha}$ в корреляционной функции полей материи имеют параметры $\alpha_{1}, \alpha_{2}$ и $\alpha_{3}$, принимающие невырожденные значения общего положения.

\section{3. ВЫСШИЕ УРАВНЕНИЯ ДВИЖЕНИЯ}

Пусть $a_{m, n}=Q / 2-\lambda_{m, n}$, где пара $(m, n)$ содержит два натуральных числа, при этом величины $V_{m, n}=V_{a_{m, n}}$ представляют собой лиувиллевские экспоненты, отвечающие вырожденным представлениям лиувиллевской алгебры Вирасоро. Пусть также $D_{m, n}^{(\mathrm{L})}$ задают соответствующие операторы "рождения сингулярных векторов", составленные из лиувиллевских генераторов алгебры Вирасоро $L_{n}$ и имеющие ту же природу, что и операторы $D_{m, n}^{(\mathrm{M})}$, введенные выше. На самом деле, операторы $D_{m, n}^{(\mathrm{L})}$ можно получить из $D_{m, n}^{(\mathrm{M})}$ с помощью подстановок $M_{n} \rightarrow L_{n}$ и $b^{2} \rightarrow-b^{2}$. Как и в ОММ, соответствующие сингулярные состояния тождественно равны нулю в ТПЛ [8]:

$$
D_{m, n}^{(\mathrm{L})} V_{m, n}=\bar{D}_{m, n}^{(\mathrm{L})} V_{m, n}=0 .
$$

Введем нормировку операторов $D_{m, n}^{(\mathrm{L})}$, которая имеет тот же вид, что и $(2.9)$,

$$
D_{m, n}^{(\mathrm{L})}=L_{-1}^{m n}+d_{1}^{(m, n)}\left(-b^{2}\right) L_{-2} L_{-1}^{m n-2}+\cdots .
$$

Также определим “логарифмически вырожденные" поля

$$
V_{m, n}^{\prime}=\left.\frac{1}{2} \frac{\partial}{\partial a} V_{a}\right|_{a=a_{m, n}}
$$

2) Заметим, что это не просто означает, что соответствующая размерность $\Delta_{1}^{(\mathrm{M})}=\alpha_{1}\left(\alpha_{1}-q\right)$ лежит в спектре Каца размерностей вырождений. Кроме этого, должны выполняться определенные соотношения между двумя другими параметрами $\alpha_{2}$ и $\alpha_{3}$, что должно обеспечить равенство нулю соответствующего сингулярного вектора. 
для каждой пары натуральных чисел $(m, n)$. Эти поля не являются примарными. Их закон преобразования при конформных преобразованиях $x \rightarrow y$ имеет вид

$$
\left|y_{x}\right|^{2 \Delta_{m, n}} V_{m, n}^{\prime}(y)=V_{m, n}^{\prime}(x)-\Delta_{m, n}^{\prime} V_{m, n}(x) \ln \left|y_{x}\right|
$$

где через $y_{x}$ обозначена производная $\partial y / \partial x$. Тем не менее, как показано в работе [5], $D_{m, n}^{(\mathrm{L})} \bar{D}_{m, n}^{(\mathrm{L})} V_{m, n}^{\prime}$ представляет собой примарное поле и, более того, операторные поля в ТПЛ удовлетворяют следующему тождеству:

$$
D_{m, n}^{(\mathrm{L})} \bar{D}_{m, n}^{(\mathrm{L})} V_{m, n}^{\prime}=B_{m, n} \widetilde{V}_{m, n},
$$

где $\widetilde{V}_{m, n}=\left.V_{a}\right|_{a=a_{m,-n}}-$ лиувиллевское экспоненциальное поле размерности $\Delta_{m, n}^{(\mathrm{L})}+$ $m n$. Числовая константа $B_{m, n}$ имеет вид

$$
B_{m, n}=\frac{\left(\pi \mu \gamma\left(b^{2}\right)\right)^{n} b^{1+2 n-2 m}}{\gamma\left(1-m+n b^{2}\right)} \prod_{k, l}^{\{m, n\}} 2 \lambda_{k, l},
$$

где через $\prod_{k, l}^{\{m, n\}}$ обозначено произведение по парам

$$
(k, l)=(\{-m+1: 1: m-1\} \otimes\{-n+1: 1: n-1\}) \backslash(0,0) .
$$

Важно отметить, что экспоненту $\widetilde{V}_{m, n}$ в ОМГ можно естественным образом объединить с соответствующим минимальным полем материи $\Phi_{m, n}$ в единый объект - одетую $(1,1)$-форму (1.20). Из этого обстоятельства следует, что ВУД играют главную роль в интегрируемости уравнения (1.17) в МГ.

\section{4. ОБОБЩЕННАЯ МИНИМАЛЬНАЯ ГРАВИТАЦИЯ}

Приведем некоторые известные результаты теории ОМГ. Как неоднократно отмечалось в литературе, параметр материи ОММ $b$ совпадает в ОМГ с параметром соответствующей ТПЛ. Именно это обстоятельство оправдывает то, что мы придерживаемся этого обозначения всюду в данной работе. Уравнение (2.2) допускает два решения относительно $\alpha$ в случае одетых полей материи $U_{a}=\Phi_{\alpha} V_{a}$. Для определенности выберем

$$
U_{a}=\Phi_{a-b} V_{a}
$$

Задача в ОМГ состоит в вычислении гравитационных корреляционных функций (1.15), в которых вклад материи задается выражениями ОММ. Таким образом, мы ограничиваемся рассмотрением таких теорий ОМГ, в которых корреляционная функция ОММ оказывается однозначно определенной.

Можно легко вычислить трехточечную функцию, если умножить $C_{\mathrm{M}}\left(a_{1}-b, a_{2}-\right.$

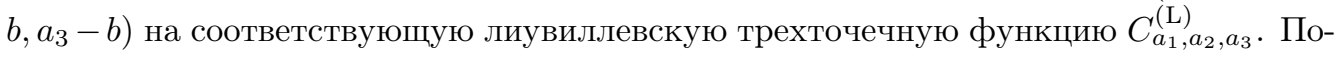
лученное произведение можно записать в виде

$$
\left\langle W_{a_{1}} W_{a_{2}} W_{a_{2}}\right\rangle_{\mathrm{OM} \Gamma}=\Omega N\left(a_{1}\right) N\left(a_{2}\right) N\left(a_{3}\right),
$$


где $W_{a}=C \bar{C} U_{a}^{3)}$,

$$
\Omega=-\left[\pi \mu \gamma\left(b^{2}\right)\right]^{Q / b}\left[\gamma\left(b^{2}\right) \gamma\left(b^{-2}-1\right) b^{-2}\right]^{1 / 2},
$$

а “факторы внешних линий” $N(a)$ имеют вид

$$
N(a)=\left[\pi \mu \gamma\left(b^{2}\right)\right]^{-a / b}\left[\gamma\left(2 a b-b^{2}\right) \gamma\left(2 a b^{-1}-b^{-2}\right)\right]^{1 / 2} .
$$

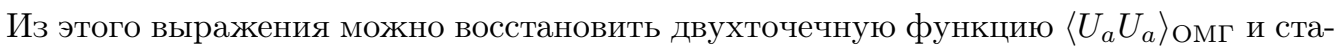
тистическую сумму $Z_{\mathrm{L}}$ :

$$
\begin{gathered}
\left\langle U_{a} U_{a}\right\rangle_{\mathrm{OM} \Gamma}=\left[\pi \mu \gamma\left(b^{2}\right)\right]^{Q / b} \frac{N^{2}(a)}{\pi(2 a-Q)}, \\
Z_{\mathrm{L}}=\left[\pi \mu \gamma\left(b^{2}\right)\right]^{Q / b} \frac{1-b^{2}}{\pi^{3} Q \gamma\left(b^{2}\right) \gamma\left(b^{-2}\right)} .
\end{gathered}
$$

Для нормированных корреляционных функций $\left\langle\left\langle U_{a} U_{a}\right\rangle\right\rangle=Z_{\mathrm{L}}^{-1}\left\langle U_{a} U_{a}\right\rangle_{\text {Омг и }}$

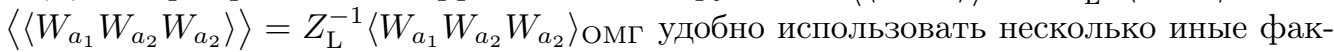
торы внешних линий

$$
\mathcal{N}(a)=\pi N(a)\left[\frac{\gamma\left(b^{2}\right) \gamma\left(b^{-2}\right)}{-\left(1-b^{-2}\right)^{2}}\right]^{1 / 2}=\frac{\pi}{(\pi \mu)^{a / b}}\left[\frac{\gamma\left(2 a b-b^{2}\right) \gamma\left(2 a b^{-1}-b^{-2}\right)}{\gamma^{2 a / b-1}\left(b^{2}\right) \gamma\left(2-b^{-2}\right)}\right]
$$

где для определенности предполагается, что ветвь квадратного корня выбрана так, чтобы обеспечить равенства

$$
\begin{gathered}
\mathcal{N}(b)=\mu^{-1}, \\
\left\langle\left\langle W_{a_{1}} W_{a_{2}} W_{a_{2}}\right\rangle\right\rangle=-\left(1+b^{-2}\right) b^{-2}\left(b^{-2}-1\right) \prod_{i=1}^{3} \mathcal{N}\left(a_{i}\right), \\
\left\langle\left\langle U_{a} U_{a}\right\rangle\right\rangle=\frac{\left(b^{-2}+1\right) b^{-2}\left(b^{-2}-1\right)}{\left(2 a b^{-1}-b^{-2}-1\right)} \mathcal{N}^{2}(a) .
\end{gathered}
$$

В случае параметра а общего положения оказалось удобным определить перенормированные поля

$$
\mathcal{U}(a)=\mathcal{N}^{-1}(a) U_{a}, \quad \mathcal{W}(a)=\mathcal{N}^{-1}(a) W_{a},
$$

для которых соотношения (4.9) принимают вид

$$
\begin{aligned}
\langle\langle\mathcal{U}(a) \mathcal{U}(a)\rangle\rangle & =\frac{(g+1) g(g-1)}{(2 s-g-1)}, \\
\left\langle\left\langle\mathcal{W}\left(a_{1}\right) \mathcal{W}\left(a_{2}\right) \mathcal{W}\left(a_{3}\right)\right\rangle\right\rangle & =-(g+1) g(g-1),
\end{aligned}
$$

где $s=a b^{-1}$ и $g=b^{-2}$. Можно явно проверить, что формально $\mathcal{W}(a)=\mathcal{W}(Q-a)$, т.е. одетые поля материи не зависят от выбора процедуры одевания в данной нормировке. Это может показаться важным преимуществом, но цена, которую приходится заплатить за него, состоит в том, что при этом факторы внешних линий (4.4) становятся сингулярными в некоторых точках (и в любом случае приобретают зависимость от космологической постоянной $\mu$ ).

\footnotetext{
3) В дальнейшем используются менее компактные обозначения $U(a)=U_{a}$ и $W(a)=W_{a}$.
} 


\section{5. ДИСКРЕТНЫЕ СОСТОЯНИЯ И ЧЕТЫРЕХТОЧЕЧНЫЙ ИНТЕГРАЛ}

Вычисление четырехточечного корреляционного числа $\left\langle U_{a_{1}} U_{a_{2}} U_{a_{3}} U_{a_{4}}\right\rangle_{\text {омг }}$, заданного интегралом (1.17), представляет собой задачу следующего уровня сложности. Если одно из полей материи оказывается вырожденным, т.е. $\Phi_{\alpha_{4}}=\Phi_{m, n}$, то четырехточечную функцию материи можно построить в явном виде с помощью соотношения (2.19). Пусть остальные три поля остаются формальными примарными полями ОММ в ситуации общего положения ${ }^{4)}$. Наша цель состоит в вычислении интеграла

$$
\left\langle U_{m, n} U_{a_{1}} U_{a_{2}} U_{a_{3}}\right\rangle_{\mathrm{OM} \Gamma}=\int\left\langle U_{m, n}(x) W_{a_{1}}\left(x_{1}\right) W_{a_{2}}\left(x_{2}\right) W_{a_{3}}\left(x_{3}\right)\right\rangle d^{2} x,
$$

в котором $U_{m, n}$ - одетое вырожденное поле $\Phi_{m, n}$, задаваемое выражением (1.20). Определим через

$$
\Theta_{m, n}=\Phi_{m, n} V_{m, n}
$$

прямое произведение вырожденных полей материи и полей Лиувилля и введем операторы

$$
\mathcal{D}_{m, n}=D_{m, n}^{(\mathrm{M})}+(-1)^{m n} D_{m, n}^{(\mathrm{L})}
$$

(и аналогично для $\left.\overline{\mathcal{D}}_{m, n}\right)$, где $D_{m, n}^{(\mathrm{M})}$ и $D_{m, n}^{(\mathrm{L})}$ представляют собой операторы "рождения сингулярных векторов" (2.9) и (3.2) для материи и для поля Лиувилля.

ПреДлОЖЕНИЕ 1. Для каждой пары $(m, n)$ положительных иелых чисел сущеcтвует оператор $H_{m, n}$, составленный из генераторов алгебр Вирасоро $M_{n} u L_{n} u$ из духовых полей $B$ и $C$. Этот оператор имеет вид градуированного многочлена степени тn-1 и духового числа 0 , причем $H_{m, n} \Theta_{m, n}$ замкнуто, но нетривиально. Оператор $H_{m, n}$ задан однозначно с точностъю до точных членов, т.е. представляет собой одномерный класс когомологий.

Это предложение можно проверить с помощью явного вычисления на нескольких первых уровнях. Имеем

$$
\begin{aligned}
H_{1,2}= & M_{-1}-L_{-1}+b^{2} C B \\
H_{1,3}= & M_{-1}^{2}-M_{-1} L_{-1}+L_{-1}^{2}-2 b^{2}\left(M_{-2}+L_{-2}\right)+ \\
& \quad+2 b^{2}\left(M_{-1}-L_{-1}\right) C B-4 b^{4} C \partial B .
\end{aligned}
$$

Доказательство для серии $(1, n)$, основанное на использовании явных выражений для операторов $D_{m, n}^{(\mathrm{L})}$ и $D_{m, n}^{(\mathrm{M})}$ [9], было приведено в работе [10]. Скорее всего, это утверждение также справедливо и в случае общих $(m, n)$ [11].

Классы когомологий $H_{m, n} \Theta_{m, n}$ были открыты в работах [12], [13], где они получили название “дискретных состояний”. Хотя общий вид операторов $H_{m, n}$ нам

\footnotetext{
4) Как обсуждалось выше, последнее требование существенно, так как в некоторых случаях корреляционные функции вырожденных полей не могут быть получены из соответствующих функций полей общего положения при непосредственном взятии предела по некоторым параметрам.
} 
неизвестен, мы будем предполагать, что их нормировка фиксирована следующим образом:

$$
H_{m, n}=\sum_{k=0}^{m n-1}\left(M_{-1}\right)^{m n-1-k}\left(-L_{-1}\right)^{k}+\cdots
$$

Очевидно, что

$$
\left(\partial H_{m, n}-\mathcal{Q} R_{m, n}\right) \Theta_{m, n}=\left(\bar{\partial} \bar{H}_{m, n}-\overline{\mathcal{Q}}_{m, n}\right) \Theta_{m, n}=0
$$

где $R_{m, n}$ снова представляет собой градуированный многочлен от генераторов $M_{n}$ и $L_{n}$ и духовых полей.

ПРЕДЛОЖЕНИЕ 2. Имеет место равенство

$$
\mathcal{D}_{m, n} \overline{\mathcal{D}}_{m, n} \Theta_{m, n}^{\prime}=\left(\partial H_{m, n}-\mathcal{Q} R_{m, n}\right)\left(\bar{\partial}_{H_{m, n}}-\overline{\mathcal{Q} R}_{m, n}\right) \Theta_{m, n}^{\prime}
$$

где

$$
\Theta_{m, n}^{\prime}=\Phi_{m, n} V_{m, n}^{\prime}
$$

u вершинный оператор $V_{m, n}^{\prime}$ задается уравнением (3.3).

Мы явно проверили соотношение $(5.7)$ для $(m, n)=(1,2)$ и $(1,3)$. Возможно, что в общем случае требуются некоторые модификации. В совокупности с ВУД (3.5) это утверждение задает точный локальный вид “когомологических ВУД” (1.22). В частности, оно позволяет заменить уравнение (5.1) уравнением (1.21) и затем переписать последнее в виде

$$
\left\langle U_{m, n} U_{a_{1}} U_{a_{2}} U_{a_{3}}\right\rangle_{\mathrm{OM} \Gamma}=B_{m, n}^{-1} \int_{\partial \Gamma} \partial\left\langle O_{m, n}^{\prime}(x) W_{a_{1}}\left(x_{1}\right) W_{a_{2}}\left(x_{2}\right) W_{a_{3}}\left(x_{3}\right)\right\rangle \frac{d x}{2 i},
$$

где

$$
O_{m, n}^{\prime}=H_{m, n} \bar{H}_{m, n} \Theta_{m, n}^{\prime} .
$$

Интеграл по пространству модулей при этом сводится к граничному интегралу и к так называемому вкладу от кривизны. Граница представляет собой три инфинитезимальных окружности $\partial \Gamma=\sum_{i=1}^{3} \partial \Gamma_{i}$ вокруг вставок $W$-полей (с интегрированием по часовой стрелке) и окружность большого радиуса $\partial \Gamma_{\infty}$ вокруг бесконечности (с интегрированием против часовой стрелки), интегрирование по которой дает член, называемый вкладом от кривизны. Чтобы вычислить граничные члены, необходимо хорошо понимать поведение операторного произведения $O_{m, n}^{\prime}(x) W_{a}(0)$ на малых расстояниях. На первом этапе обсудим член, задающий вклад от кривизны.

\section{6. ВКЛАД ОТ КРИВИЗНЫ}

Член, учитывающий вклад от кривизны, возникает ввиду того, что оператор $O_{m, n}^{\prime}$ не является в точности скаляром (т.е. $(0,0)$-формой), но представляет собой логарифмическое поле. При конформных преобразованиях координат $x \rightarrow y$ он приобретает неоднородную добавку:

$$
O_{m, n}^{\prime}(y)=O_{m, n}^{\prime}(x)-\Delta_{m, n}^{\prime} O_{m, n}(x) \ln \left|y_{x}\right|,
$$


где

$$
O_{m, n}=H_{m, n} \bar{H}_{m, n} \Theta_{m, n}
$$

- элемент кольца дискретных состояний (см. ниже) и

$$
\Delta_{m, n}^{\prime}=\left.\frac{d}{d a} \Delta_{a}^{(\mathrm{L})}\right|_{a=a_{m, n}}=m b^{-1}+n b=2 \lambda_{m, n}
$$

Можно учесть это обстоятельство двумя возможными способами. Во-первых, легко показать, что преобразование (6.1) на сфере приводит к следующему поведению корреляционной функции с $O_{m, n}^{\prime}(x)$ при $x \rightarrow \infty$ :

$$
\left\langle O_{m, n}^{\prime}(x) W_{a_{1}}\left(x_{1}\right) W_{a_{2}}\left(x_{2}\right) W_{a_{3}}\left(x_{3}\right)\right\rangle \sim-\Delta_{m, n}^{\prime} \ln (x \bar{x})\left\langle O_{m, n} W_{a_{1}} W_{a_{2}} W_{a_{3}}\right\rangle .
$$

Таким образом, вклад от кривизны можно включить как граничный член $\partial \Gamma_{\infty}$, отвечающий контуру вокруг бесконечной точки. Этот член можно вычислить в следующем виде:

$$
\frac{1}{2 i} \int_{\partial \Gamma_{\infty}} \partial\left\langle O_{m, n}^{\prime}(x) W_{a_{1}}\left(x_{1}\right) W_{a_{2}}\left(x_{2}\right) W_{a_{3}}\left(x_{3}\right)\right\rangle d x=-2 \pi \lambda_{m, n}\left\langle O_{m, n} W_{a_{1}} W_{a_{2}} W_{a_{3}}\right\rangle .
$$

Во-вторых, существует прием, который легче обобщить на случай поверхностей с более сложной геометрией. Он состоит в том, что мы сохраняем след фоновой метрики $\hat{g}_{a b}=e^{\sigma} \delta_{a b}$. Поскольку закон преобразования масштабного множителя $\sigma(x)$ при конформных преобразованиях имеет вид

$$
\sigma(y)=\sigma(x)-2 \ln \left|y_{x}\right|
$$

комбинация полей

$$
\widetilde{O}_{m, n}^{\prime}(x)=O_{m, n}^{\prime}(x)-\frac{\Delta_{m, n}^{\prime} \sigma(x) O_{m, n}(x)}{2}
$$

оказывается скаляром (это достигается ценой введения зависимости от фоновой метрики). Таким образом, в БРСТ-инвариантной формулировке, формула (5.7) может быть переписана в виде

$$
B_{m, n} U_{m, n}=\sqrt{\hat{g}}\left(\frac{1}{4} \hat{\Delta} \widetilde{O}_{m, n}^{\prime}-\frac{\Delta_{m, n}^{\prime}}{8} \widehat{R} O_{m, n}\right)
$$

где $\hat{\Delta}$ - оператор Лапласа, ковариантный по отношению к $\hat{g}_{a b}$, а $\widehat{R}$ - соответствующая скалярная кривизна. На сфере вклад второго члена очевидно сводится к выражению (6.5).

На этом этапе становится ясным, что правильное понимание структуры кольца дискретных состояний в ОМГ, в частности, вычисление вакуумного ожидания в правой части равенства (6.5), весьма важно для воплощения нашей программы вычислений. 


\section{7. КОЛЬЦО ДИСКРЕТНЫХ СОСТОЯНИЙ В ОМГ}

В работах [12], [13] было обнаружено, что в теории МГ вырожденные поля $\Phi_{m, n}$ ОММ в комбинации с вырожденными показателями $V_{m, n}$ соответствующей ТПЛ задают нетривиальные БРСТ-инвариантные операторы (6.2) с нулевым духовым числом и конформной размерностью $(0,0)$. Некоторые из этих операторов были явно вычислены в работе [10]. Пространственные производные $\partial O_{m, n}$ и $\bar{\partial} O_{m, n}$ оказываются БРСТ-точными (5.6). Таким образом, в БРСТ-замкнутом секторе корреляционные функции этих дискретных состояний не зависят от координат вставок этих операторов. Более того, эти операторы, рассматриваемые как классы БРСТкогомологий, образуют замкнутое кольцо по отношению к ОР, называемое кольцом дискретных состояний (КДС). Это наблюдение позволило Виттену [13] заключить, что указанный объект играет ключевую роль в МГ и что полная алгебраическая структура теории, может быть, совпадает со структурой КДС. В этом разделе будут представлены некоторые явные вычисления, демонстрирующие свойства КДС. Когомологические свойства операторов $O_{m, n}$ проявляются только в $\mathcal{Q}$-инвариантном описании. Простейшее инвариантное состояние на сфере создается тремя операторами $W_{a}$. По этой причине мы представим явные вычисления для корреляционной функции $\left\langle O_{m, n} W_{a_{1}} W_{a_{2}} W_{a_{3}}\right\rangle$ на сфере с тремя вставками $W_{a}$ общего вида. Заметим, что опять предполагается, что все три параметра $a_{1}, a_{2}$ и $a_{3}$ находятся в общем положении. Если одно или более из полей $W_{a}$ содержит приводимые представления полей материи или лиувиллевской алгебры Вирасоро, то могут возникнуть всевозможные тонкие эффекты, которые мы не намерены обсуждать в данной работе.

Дискретные состояния $O_{m, n}$ действуют в пространстве классов $W_{a}$ по модулю точных форм. Это является следствием того, что их действие не меняет духовое число, и все нетривиальные классы в ситуации общего положения исчерпываются композитными полями $W_{a}$ с различными $a$. Более того, благодаря ограничениям на ОР для вырожденных полей $\Phi_{m, n}$ и $V_{m, n}$ с соответствующими примарными полями $\Phi_{\alpha}$ и $V_{a}$ общая структура операторного произведения $O_{m, n}(x) W(a)$ обречена иметь вид

$$
O_{m, n} W(a)=\sum_{r, s=0}^{(m, n)} A_{r, s}^{(m, n)} W\left(a+\lambda_{r, s}\right)+\text { точные члены }
$$

с некоторыми числовыми коэффициентами $A_{r, s}^{(m, n)}$. Наша непосредственная задача состоит в вычислении этих чисел.

Полезно сначала произвести явное вычисление в простейшем случае $(m, n)=$ $(1,2)$. Специальные ОР, которые нам необходимы в этом случае, задаются формулой (2.13) и соотношением

$$
V_{1,2}(y) V_{a}(0)=C_{+}^{(\mathrm{L})}(a)(y \bar{y})^{a b}\left[V_{a-b / 2}\right]+C_{-}^{(\mathrm{L})}(a)(y \bar{y})^{1-a b+b^{2}}\left[V_{a+b / 2}\right],
$$

где

$$
C_{+}^{(\mathrm{L})}(a)=1, \quad C_{-}^{(\mathrm{L})}(a)=-\frac{\pi \mu}{\gamma\left(-b^{2}\right)} \frac{\gamma\left(2 a b-b^{2}-1\right)}{\gamma(2 a b)} .
$$


Явными вычислениями легко проверить (по крайней мере, на уровне примарных полей), что в произведении $U_{a}=\Phi_{a-b} V_{a}$ действие операторов $H_{1,2}$ и $\bar{H}_{1,2}$ устраняет "нежелательные члены" (т.е. члены, содержащие комбинации $\Phi_{a-b / 2} V_{a-b / 2}$ и $\left.\Phi_{a-3 b / 2} V_{a+b / 2}\right)$ и остаются только ${ }^{5)}$

$$
O_{1,2} W(a)=A_{0,-1}^{(1,2)} W\left(a-\frac{b}{2}\right)+A_{0,1}^{(1,2)} W\left(a+\frac{b}{2}\right)+\text { точные члены, }
$$

где в явном виде

$$
\begin{aligned}
& A_{0,-1}^{(1,2)}=\left(1-2 a b+b^{2}\right)^{2} C_{-}^{(\mathrm{M})}(a-b) C_{+}^{(\mathrm{L})}(a), \\
& A_{0,1}^{(1,2)}=\left(1-2 a b+b^{2}\right)^{2} C_{+}^{(\mathrm{M})}(a-b) C_{-}^{(\mathrm{L})}(a) .
\end{aligned}
$$

Полиномиальные множители в формулах (7.5) появляются в результате действия $H_{1,2} \bar{H}_{1,2}$ на соответствующие члены в разложении $\Theta_{1,2}(x) W_{a}(0)$. Аналогичные вычисления могут быть проведены непосредственно для действия каждого оператора $\Theta_{m, n}$, уровень за уровнем. Нами было явно проверено сокращение “нежелательных членов" в случае $(m, n)=(1,3)$ и найдены многочлены, возникающие вследствие действия $H_{1,3} \bar{H}_{1,3}$. Ответ имеет следующий вид:

$$
N\left(a+\lambda_{r, s}\right) A_{r, s}^{(m, n)}=\Lambda_{m, n} N(a),
$$

где

$$
\pi \Lambda_{m, n}=B_{m, n} \mathcal{N}\left(a_{m,-n}\right)
$$

и $B_{m, n}$ - те же, что и в равенстве (3.6), а множитель $\mathcal{N}(a)$ был введен в формуле (4.7).

Представляется желательным упростить эти соотношения с помощью введения перенормированных полей $\mathcal{W}(a)$, как это было сделано в формуле $(4.10)$, и оператоpoв

$$
\mathcal{O}_{m, n}=\Lambda_{m, n}^{-1} O_{m, n}
$$

Тогда выражение (7.1) сводится к формуле

$$
\mathcal{O}_{m, n} \mathcal{W}(a)=\sum_{k, l}^{(m, n)} \mathcal{W}\left(a+k b+l b^{-1}\right) .
$$

Это выражение совпадает с полученным ранее в работах [15] на основе более общих предположений. Здесь мы получаем то же выражение с помощью прямых вычислений [16] (см. также соответствующие исследования в работах [14], [17]). Пока нами явно было проверено лишь ограниченное число частных примеров. В частности, простое выражение (7.6) появляется в результате загадочных соотношений между различными членами в явных выражениях для $H_{m, n}$ и для сингулярных векторов. Сложные вычисления приводят к простым результатам, и это, очевидно, свидетельствует о наличии скрытой структуры. Однако проблема построения

5) Другой способ вычисления действия $O_{1,2}$ на общий класс $W(a)$ был применен в работе [14]. Насколько нам известно, Петкова пришла к этому выводу весной 2004 г. Более раннее обсуждение этой задачи содержится в работах [15]. 
общего вывода, открывающего эту структуру, остается открытой. Другое важное отличие нашего рассмотрения от подхода работ [15] состоит в том, что мы рассматривали действие $O_{m, n}$ на когомологии $W_{a}$ с общим $a$. Естественно ожидать, что соотношения (7.9) модифицируются, если их применить к вырожденным классам $W_{m, n}=C \bar{C} \Phi_{m, n} \widetilde{V}_{m, n}$, отвечающим неприводимым представлениям симметрии Вирасоро полей материи (т.е. при нулевых сингулярных векторах в секторе материи). Хотя этот эффект может просто привести к правильному обрыву суммы в формуле (7.9), следующему из алгебры слияния для вырожденных полей, предел $a \rightarrow a_{m,-n}$ в этом выражении достаточно технически сложен и требует более тщательного исследования. Таким образом, как было отмечено ранее, в данной работе мы ограничиваем рассмотрение случаем общих значений параметра $a$, откладывая ситуацию вырожденных неприводимых полей для последующих исследований. Наш результат оказывается достаточным для последующего исследования интеграла (5.1) с тремя общими невырожденными значениями параметров $a_{1}, a_{2}$ и $a_{3}$.

Из простого вида формулы (7.9) естественно вытекает следующая структура алгебры КДС:

$$
\mathcal{O}_{m, n} \mathcal{O}_{m^{\prime}, n^{\prime}}=\sum_{l}^{\left[m, m^{\prime}\right]} \sum_{k}^{\left[n, n^{\prime}\right]} \mathcal{O}_{l, k}
$$

где символ $\sum_{k}^{\left[n, n^{\prime}\right]}$ обозначает сумму по $k=\left\{\left|n-n^{\prime}\right|+1: 2: n+n^{\prime}-1\right\}$. Следуя работам [15], можно ввести производящие элементы $X=\mathcal{O}_{1,2} / 2, \quad Y=\mathcal{O}_{2,1} / 2$ и переписать формулу (7.10) в виде

$$
\mathcal{O}_{m, n}=U_{m-1}(Y) U_{n-1}(X)
$$

где $U_{n}(x)$ - многочлены Чебышева второго рода.

\section{8. ГРАНИЧНЫЕ ЧЛЕНЫ}

Три интеграла по границе $\partial \Gamma=\sum_{i=1}^{3} \partial \Gamma_{i}$ в формуле $(5.9)$,

$$
g_{i}=\int_{\partial \Gamma_{i}} \partial\left\langle O_{m, n}^{\prime}(x) W_{a_{1}}\left(x_{1}\right) W_{a_{2}}\left(x_{2}\right) W_{a_{3}}\left(x_{3}\right)\right\rangle \frac{d x}{2 i},
$$

контролируются ОР “логарифмического примарного” поля $O_{m, n}^{\prime}$ и состояний (общего положения в нашем случае) $W\left(a_{i}\right)$. Прямой способ вычислить это разложение состоит в том, чтобы выполнить сначала разложение для произведения "первообразных" $\Theta_{m, n}^{\prime}(x)=\Phi_{m, n}(x) V_{m, n}^{\prime}(x)$, а затем одеть их с помощью $C \bar{C}(0)$ и применить $H_{m, n} \bar{H}_{m, n}$. Последнее ОР снова представляет собой произведение двух независимых разложений для $\Phi_{m, n}(x) \Phi_{\alpha}(0)$ и для $V_{m, n}^{\prime}(x) V_{a}(0)$. В то время как первое разложение представляет собой то же дискретное вырожденное ОР (2.15), что и в случае вычисления в КДС, второе оказывается более сложным и требует отдельного исследования. 
Наиболее прямой способ вычислить его - это начать с общего "непрерывного" OP (1.10), которое мы здесь запишем как ${ }^{6)}$

$$
V_{g}(x) V_{a}(0)=\int_{\uparrow}^{\prime} \frac{d p}{4 \pi i} C_{g, a}^{(\mathrm{L}) p}(x \bar{x})^{\Delta_{p}^{(\mathrm{L})}-\Delta_{g}^{(\mathrm{L})}-\Delta_{a}^{(\mathrm{L})}}\left[V_{p}(0)\right],
$$

где контур $\uparrow$ проходит через $Q / 2$ вдоль мнимой оси, а штрих обозначает деформации, необходимые для аналитического продолжения из “главной области" (1.11). Сингулярности структурной константы

$$
C_{g, a}^{(\mathrm{L}) p}=\frac{\left(\pi \mu \gamma\left(b^{2}\right) b^{2-2 b^{2}}\right)^{(p-a-g)} \Upsilon_{b}(b) \Upsilon_{b}(2 g) \Upsilon_{b}(2 a) \Upsilon_{b}(2 Q-2 p)}{\Upsilon_{b}(p+a-g) \Upsilon_{b}(a+g+p-Q) \Upsilon_{b}(a+g-p) \Upsilon_{b}(p+g-a)}
$$

задаются нулями четырех $\Upsilon_{b}$-функций в знаменателе. Пример расположения этих нулей показан на рис. 1, где оба параметра $a$ и $g$ вещественны, положительны и меньше, чем $Q / 2$. Данный рисунок соответствует "главной области", т.е. области $a+g>Q / 2$. "Правые" нули всех четырех множителей в знаменателе расположены справа, а “левые" нули - слева от контура интегрирования $\uparrow$, который в данном случае является прямой, проходящей вертикально через $Q / 2$. Серии нулей слегка отодвинуты от вещественной оси, чтобы лучше различать нули, соответствующие различным множителям. Самая верхняя и следующая за ней серия нулей отвечают множителям $\Upsilon_{b}(p+a-g)$ и $\Upsilon_{b}(a+g+p-Q)$, соответственно. За ними следуют нули, обусловленные $\Upsilon_{b}(a+g-p)$, а самая нижняя серия нулей порождена множителем $\Upsilon_{b}(p+g-a)$. Тогда если, к примеру, параметр $g$ уменьшается и $a+g$ становится

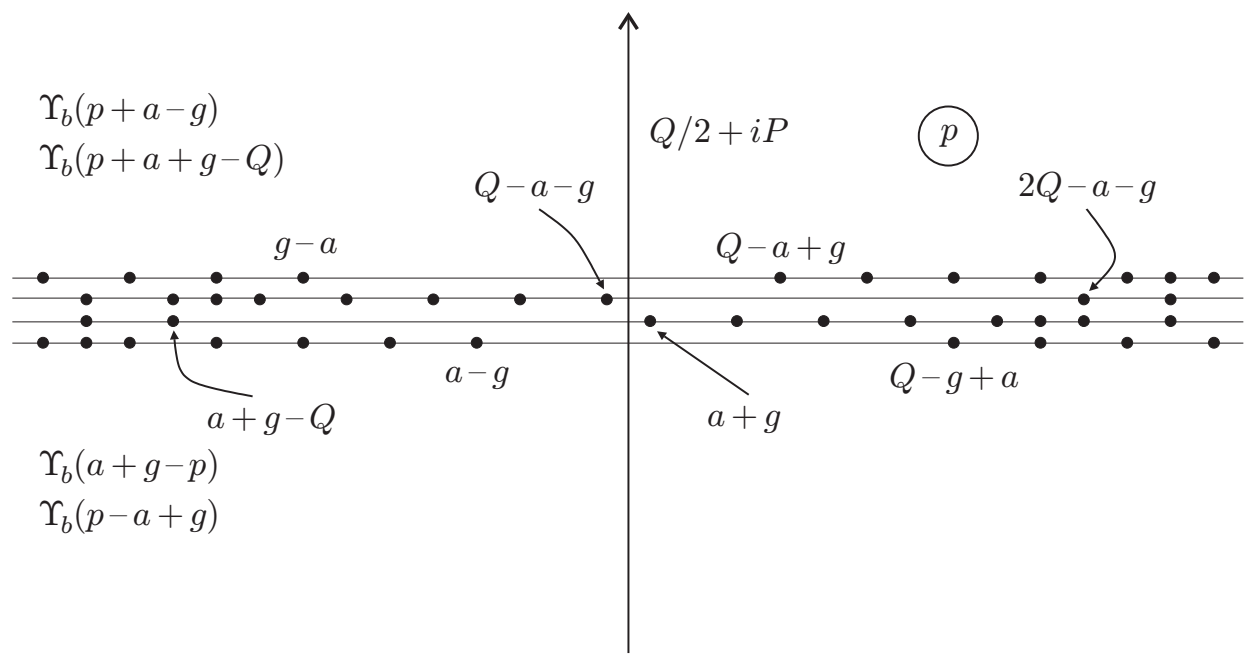

Рис. 1. Положение полюсов структурной константы при $a+g>Q / 2$.

меньше, чем $Q / 2$, то два полюса при $a+g$ и $Q-a-g$ пересекают вертикальную ось $\operatorname{Re} p=Q / 2$ (часто называемую границей Зайберга [18]). Условие аналитичности требует преобразования контура интегрирования согласованным способом (см. рис. 2).

6) В этом разделе буква $g$ не обозначает $b^{-2}$, как в разделе 4 и далее в разделе 11. 


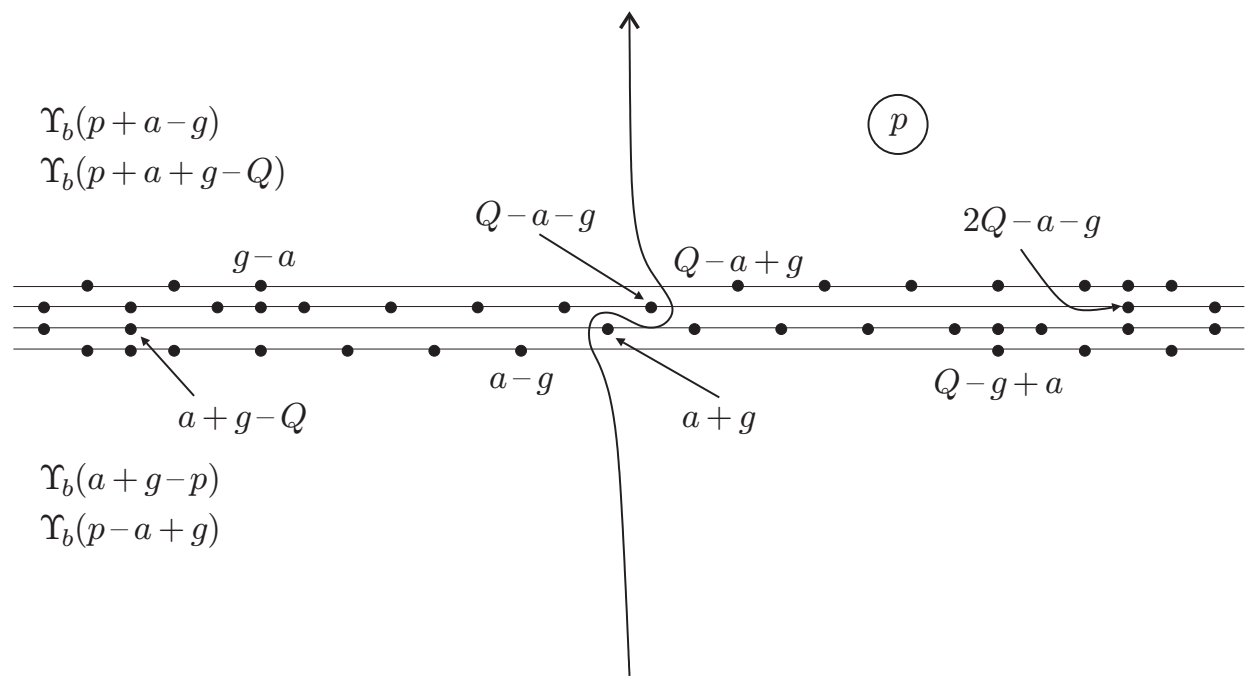

Рис. 2. Деформация контура, обусловленная аналитическим продолжением ОР (8.2) во внешность главной области.

Эффект, вызванный этой деформацией контура, может быть выделен в виде так называемых дискретных членов, как показано на рис. 3, где полюсы $a+g$ и $Q-a-g$ обведены кружочками. Вычеты в этих полюсах вычисляются явно, что приводит к формуле

$$
\begin{gathered}
V_{g}(x) V_{a}(0)=\frac{1}{2}(x \bar{x})^{-2 a g}\left[V_{a+g}(0)\right]+\frac{1}{2}(x \bar{x})^{-2 a g} R_{\mathrm{L}}(a+g)\left[V_{Q-a-g}(0)\right]+ \\
+\int_{\uparrow} \frac{d p}{4 \pi i} C_{g, a}^{(\mathrm{L}) p}(x \bar{x})^{\Delta_{p}^{(\mathrm{L})}-\Delta_{g}^{(\mathrm{L})}-\Delta_{a}^{(\mathrm{L})}}\left[V_{p}(0)\right] .
\end{gathered}
$$

Заметим, что два дискретных члена в формуле (8.4) на самом деле идентичны благодаря соотношению отражения (1.8). Это является следствием полной симметрии интеграла (8.2) при преобразовании отражения $p \rightarrow Q-p$, и это свойство, таким образом, выполняется при всех "зеркальных отражениях" относительно данной симметрии. Ниже мы используем его, чтобы держать во внимании только один из каждой пары образов, скажем тот, который отвечает $\operatorname{Re} p<Q / 2$, а затем удвоить ответ. Дальнейшее изменение параметров приводит к тому, что контур интегрирования пересекает все больше полюсов и все больше дискретных членов появляется в правой части равенства (8.4).

Сделаем еще одно важное замечание. При выводе формулы (8.4) мы предполагали, что $\operatorname{Re}(a+g)<Q / 2$. Рассматривая противоположный случай $\operatorname{Re}(a+g)>Q / 2$, сразу получим, что нам необходимо выбрать полюсы $p=Q-a+g$ и $p=a-g$. Это приводит к тому, что уравнение (8.4) заменяется на

$$
V_{g}(x) V_{a}(0)=(x \bar{x})^{-2(Q-a) g} R_{\mathrm{L}}(a)\left[V_{Q-a+g}(0)\right]+\int_{\uparrow} \frac{d p}{4 \pi i} C_{g, a}^{(\mathrm{L}) p}(x \bar{x})^{\Delta_{p}^{(\mathrm{L})}-\Delta_{g}^{(\mathrm{L})}-\Delta_{a}^{(\mathrm{L})}}\left[V_{p}(0)\right] .
$$




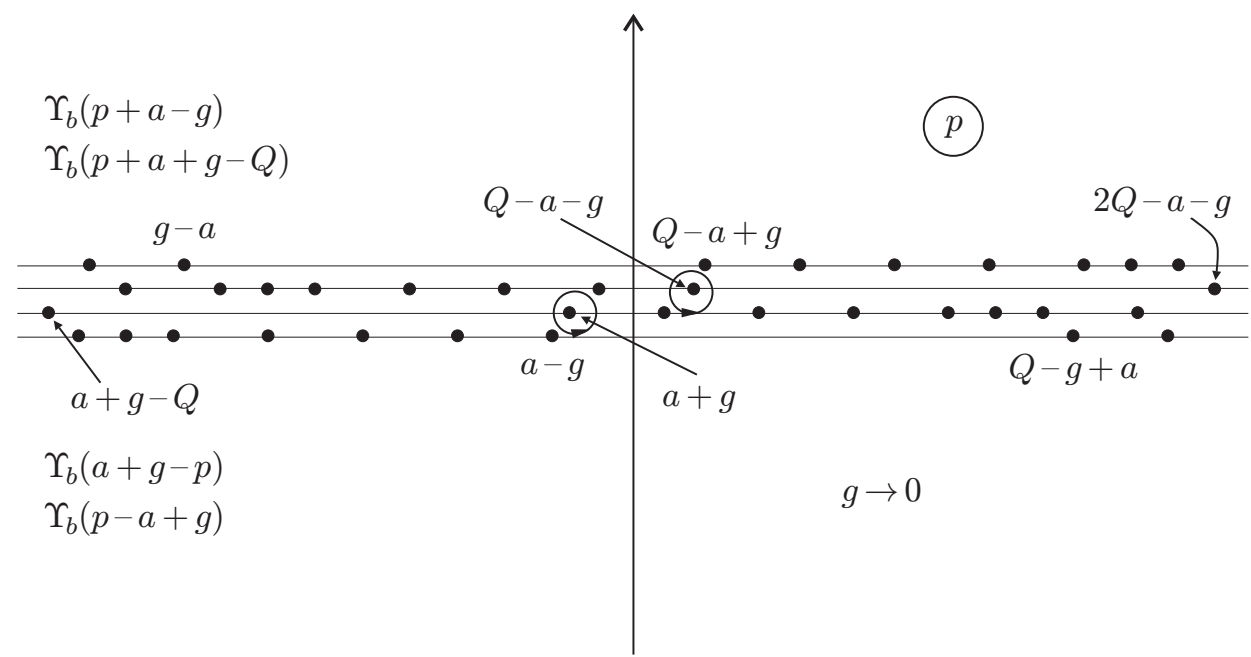

Рис. 3. "Дискретные члены”, обусловленные полюсами в точках $p=$ $a+g$ и $p=Q-a-g$, выделены явно. Эти вклады сингулярны при $g \rightarrow 0$ ввиду присутствия близких полюсов в точках $p=a-g$ и $p=Q-a+g$, соответственно. Заметим, что в качестве дискретного члена выбран полюс, расположенный вблизи вертикальной прямой $\operatorname{Re} p=Q / 2$.

Вообще, если два полюса в подынтегральном выражении сжимают контур интегрирования аналогично тому, как мы только что наблюдали в простом примере, то правильно выбирать вычет в точности в том полюсе, который оказался ближе всего к границе $\operatorname{Re} p=Q / 2$ (и затем поместить оставшийся контур интегрирования в его исходную позицию $\operatorname{Re} p=Q / 2)$. Иной выбор приводит к несогласованности, поскольку в этом случае остающаяся интегральная часть содержит более важные члены, чем те, которые уже были приняты в расчет. Наконец, оказывается удобным использовать соотношения отражения и переместить все дискретные вставки $V_{a}$ в полуплоскость $\operatorname{Re} a<Q / 2$.

Наша цель состоит в исследовании соотношения (8.2) при значениях параметра $g$, близких к определенному вырожденному значению: $g \rightarrow a_{m, n}=Q / 2-\lambda_{m, n}$. Сразу видно, что структурная постоянная (8.3) содержит общий множитель $\Upsilon_{b}(2 g)$, который обращается в нуль в этом пределе. Следовательно, сингулярности, возникающие из расходимостей интеграла, играют весьма важную роль. Чтобы понять, что происходит в общем случае, рассмотрим сначала наиболее простой из всех возможных случаев: $g \rightarrow a_{1,1}=0$. Соответствующее вырожденное поле $V_{1,1}$ представляет собой просто тождественный оператор, а логарифмическое примарное поле $V_{1,1}^{\prime}$ совпадает с основным полем Лиувилля $\phi$. В пределе $g \rightarrow 0$ интегральный член исчезает в обоих уравнениях (8.4) и $(8.5)$, и мы приходим к чистому члену $V_{a}(0)$ (что и должно быть в случае тождественного оператора вместо оператора $V_{g}$ в левой части каждого из этих уравнений). Этот случай есть тривиальный случай дискретного 
вырожденного ОР (подобно (2.15) в ОММ):

$$
V_{m, n}(x) V_{a}(0)=\sum_{r, s}^{(m, n)}(x \bar{x})^{\lambda_{r, s}\left(Q-2 a-\lambda_{r, s}\right)-\Delta_{m, n}^{(\mathrm{L})}} C_{r, s}^{(\mathrm{L})}(a)\left[V_{a+\lambda_{r, s}}\right],
$$

которое выполняется для полей $V_{m, n}$ ввиду отщепления (3.1) сингулярных векторов. Далее член, линейный по $g$, в выражении (8.4) при $g \rightarrow 0$, дает

$$
\phi(x) V_{a}(0)=-a \ln (x \bar{x}) V_{a}(0)+\text { менее сингулярные члены }
$$

Это логарифмическое $\mathrm{OP}$, которое выполняется при $\operatorname{Re} a<Q / 2$, явно напоминает схожее выражение в теории свободного скалярного поля. Однако при $\operatorname{Re} a>Q / 2$ следует вместо этого продифференцировать уравнение (8.5) по переменной $g$. Окончательный результат

$$
\phi(x) V_{a}(0)=\left(\left|\frac{Q}{2}-a\right|_{\operatorname{Re}}-\frac{Q}{2}\right) \ln (x \bar{x}) V_{a}(0)+\cdots
$$

легче сформулировать, используя обозначение

$$
|x|_{\operatorname{Re}}= \begin{cases}x, & \operatorname{Re} x>0 \\ -x, & \operatorname{Re} x<0\end{cases}
$$

которое и будет неоднократно применяться в дальнейшем. Заметим, что для логарифмически вырожденных полей интегральный член в правой части уравнения (8.5) не равен нулю, как это имеет место в случае настоящего вырожденного поля. OP (8.8) остается непрерывным, хотя интегральный член оказывается менее сингулярным, чем логарифмический ${ }^{7)}$. Как скоро станет ясным, механизм, приводящий в этом простом случае к неаналитической структуре вида (8.9) имеет общий характер и порождает все неаналитичности в граничных членах.

После этого простого "разогревающего" примера, рассмотрим более сложный случай $g \rightarrow-b$, который отвечает полю $V_{1,2}$, а в логарифмическом случае - полю $V_{1,2}^{\prime}$. Пример расположения полюсов структурной константы показан на рис. 4. Серия "правых" нулей функции $\Upsilon_{b}(a+g-p)$ проникает более глубоко в правую полуплоскость $\operatorname{Re} p<Q / 2$, и первый и второй нули при $p=a+g$ и $p=a+g+b$ стремятся соответственно ко второму и первому нулям "левой" серии нулей множителя $\Upsilon_{b}(p-a+g)$ при $p=a-g-b$ и при $p=a-g$ (как обычно, возникают симметричные относительно замены $p \rightarrow Q-p$ пинчи в левой полуплоскости $\operatorname{Re} p>Q / 2$, которые приводят к тождественным вкладам, а потому не обсуждаются по отдельности). В пределе $g \rightarrow-b$ эти пинчи порождают сингулярности, которые нейтрализуют общий нуль в множителе $\Upsilon_{b}(2 g)$ и приводят непосредственно к двухчленному дискретному ОР (7.2). Разумеется, это дискретное вырожденное ОР, как и более общее ОР (8.6), можно вывести более простым способом из условия отщепления нулевых векторов и из условий самосогласованности (условий бутстрапа). Мы тем не менее воспроизводим эти ОР более систематически из общего лиувиллевского ОР (1.10) в основном с

\footnotetext{
7)Это происходит из-за того, что мы всегда выбираем полюс, который расположен наиболее близко к прямой $\operatorname{Re} p=Q / 2$.
} 


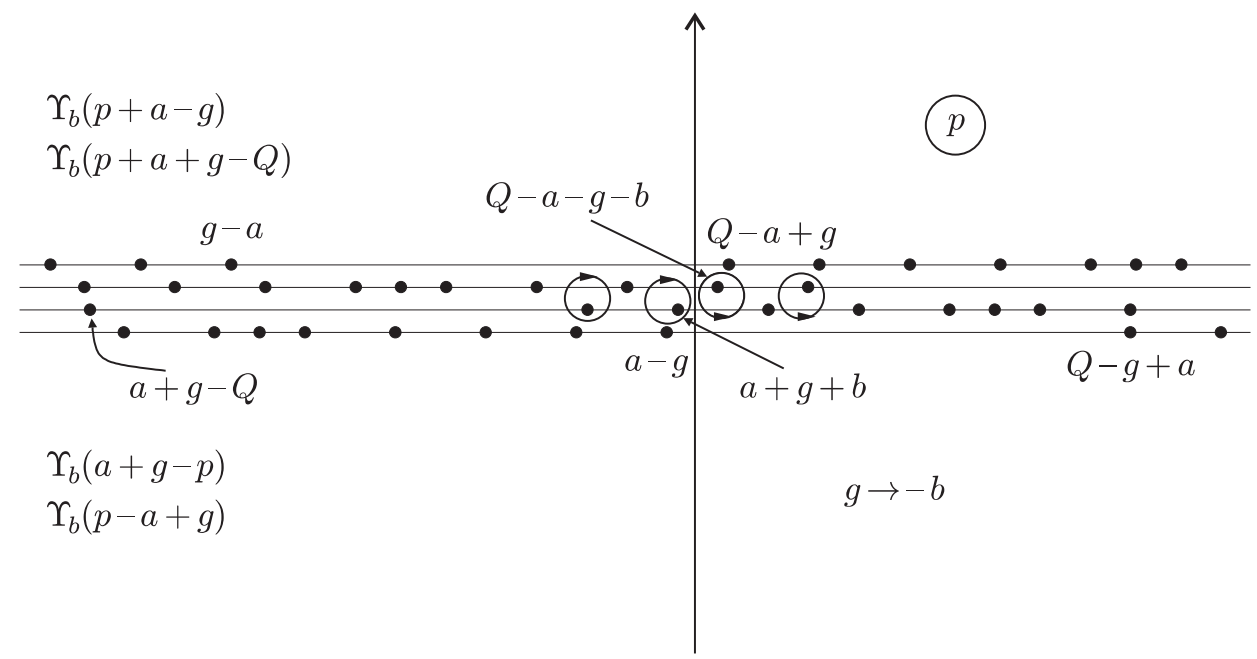

Рис. 4. Сингулярные члены в интеграле (8.2) в пределе $g \rightarrow-b$. Дискретные члены явно выделены. Эта картина отвечает случаю $\operatorname{Re}(a+g)<Q / 2-b$.

целью продемонстрировать механизм, приводящий к сингулярным дискретным членам и к обращению в нуль непрерывной интегральной составляющей. Более того, этот подход подсказывает способ найти значимые члены в OP $V_{m, n}^{\prime}(x) V_{a}(0)=\ldots$, т.е. в следующем порядке разложения по $g+b$.

Во-первых, ясно, что члены вида

$$
O_{m, n}^{\prime}(x) W_{a}(0)=\cdots+\ln (x \bar{x}) R_{m, n}^{a}(0)+\cdots
$$

(где $R_{m, n}^{a}$ - некоторый локальный оператор, обсуждаемый ниже) представляют наибольший интерес при вычислении граничных членов (8.1), поскольку

1) эти члены задают конечные вклады в (8.1);

2) менее сингулярные члены не играют роли в интеграле (8.1);

3) вклады от более сингулярных членов (если таковые имеются ${ }^{8)}$ ) сингулярно зависят от радиуса окружности $\partial \Gamma_{i}$; в полевой теории такие расходимости обычно связываются с некоторыми сингулярными перенормировками, а потому мы не учитываем их в определении интеграла (5.1).

Члены этого типа могут возникать только из тех вкладов в выражение для $V_{m, n}^{\prime}(x) V_{a}(0)$, в которых производная по $g$ в уравнении (8.2) (или по $a$ в определении (3.3)) действует на показатель степени $(x \bar{x})$. Более того, такие члены возникают только в дискретных членах, в которых нули $\Upsilon_{b}(2 g)$ компенсируются сингулярностями интеграла (в частности, они никогда не встречаются в остаточных "непрерывных" слагаемых). Краткое размышление делает очевидным следующий факт:

\footnotetext{
8) Как обсуждалось выше, остаточный интегральный член менее сингулярен, чем дискретные члены, выделяемые согласно нашему предписанию. Еще более высокие сингулярности могут возникать на коротких расстояниях как дополнительные дискретные члены, которые равны нулю в вырожденном разложении, но сохраняются в логарифмическом.
} 
интересующие нас члены - это в точности те, что возникают в дискретных ОР

$$
\begin{aligned}
V_{1,2}^{\prime}(x) V_{a}(0)= & \ln (x \bar{x})\left(q_{0,1}^{(1,2)}(a)(x \bar{x})^{a b} C_{+}^{(\mathrm{L})}(a) V_{a-b / 2}(0)+\right. \\
& \left.+q_{0,-1}^{(1,2)}(a)(x \bar{x})^{1-a b+b^{2}} C_{-}^{(\mathrm{L})}(a) V_{a+b / 2}(0)\right)+\cdots,
\end{aligned}
$$

дополненные, однако, определенными множителями

$$
q_{0, s}^{(1,2)}(a)=\left|a-\frac{b s}{2}-\frac{Q}{2}\right|_{\operatorname{Re}}-\lambda_{1,2}
$$

Эти множители возникают в результате взятия производной по $g$ от показателя степени выражения $x \bar{x}$, в то время как неаналитичность объясняемся тем, что в различных областях определения параметра $a$ в качестве дискретных членов следует выбирать различные полюсы (снова напомним, что в сливающихся парах полюсов мы всегда явно выбираем тот из них, который по переменной $p$ находится ближе к границе Зайберга $\operatorname{Re} p=Q / 2)$. Это тот же механизм неаналитичности, который наблюдался в уравнении (8.8).

Коль скоро мы нашли необходимые члены в логарифмическом лиувиллевском ОР $V_{1,2}^{\prime}(x) V_{a}(0)$, все последующие вычисления буквально повторяют те, которые использовались при выводе (7.4). Опуская прямые вычисления (которые показывают, что "нежелательные" перекрестные члены в произведении (8.1) и (2.13) исчезают после применения $H_{1,2} \bar{H}_{1,2}$, и дают снова знакомые многочлены от "хороших" членов), приведем только конечный результат, принимающий более красивый вид, если представить его через перенормированные поля $W_{a}$, как было сделано в уравнении (4.10):

$$
\begin{gathered}
O_{1,2}^{\prime}(x) \mathcal{W}_{a}=\Lambda_{1,2} \ln (x \bar{x})\left(q_{0,1}^{(1,2)}(a) \mathcal{W}_{a-b / 2}+q_{0,-1}^{(1,2)}(a) \mathcal{W}_{a+b / 2}\right)+ \\
+ \text { нелогарифмические члены. }
\end{gathered}
$$

Здесь величины $\Lambda_{1,2}$ те же, что и в уравнении (7.7).

Два примера, рассмотренные выше, прокладывают прямую дорогу к общему случаю. Необходимые логарифмические члены в $V_{m, n}^{\prime}(x) V_{a}(0)$ имеют вид

$$
V_{m, n}^{\prime}(x) V_{a}(0)=\sum_{r, s}^{(m, n)}(x \bar{x})^{\lambda_{r, s}\left(Q-2 a-\lambda_{r, s}\right)-\Delta_{m, n}^{(\mathrm{L})}} q_{r, s}^{(m, n)}(a) C_{r, s}^{(\mathrm{L})}(a)\left[V_{a+\lambda_{r, s}}\right],
$$

где выражение (8.12) обобщается до

$$
q_{r, s}^{(m, n)}(a)=\left|a-\lambda_{r, s}-\frac{Q}{2}\right|_{\operatorname{Re}}-\lambda_{m, n}
$$

а сумма снова берется по стандартному набору (2.16). Общую версию формулы (8.13) можно непосредственно получить из выражения (7.6) в разделе 7. При вычислениях в КДС это выражение не было доказано, а только угадано на основании явных ответов при $(m, n)=(1,2)$ и $(m, n)=(1,3)$. В настоящем подходе 
нет необходимости повторять вычисления в случае $(1,3)$, поскольку, как было показано выше, для логарифмических членов они в точности повторяют рассуждения раздела 7. Таким образом, из угадываемой формулы (7.6) следует, что

$$
\mathcal{O}_{m, n}^{\prime}(x) \mathcal{W}_{a}=\ln (x \bar{x}) \sum_{r, s}^{(m, n)} q_{r, s}^{(m, n)}(a) \mathcal{W}_{a-\lambda_{r, s}}
$$

где удобно избавиться от $\Lambda_{m, n}$ с помощью перенормировки вставки $O_{m, n}^{\prime}$, сходной с той, что была использована в выражениии (7.8):

$$
\mathcal{O}_{m, n}^{\prime}(x)=\Lambda_{m, n}^{-1} O_{m, n}^{\prime}
$$

\section{9. ЧЕТЫРЕХТОЧЕЧНОЕ КОРРЕЛЯЦИОННОЕ ЧИСЛО}

Теперь мы в состоянии представить главный результат данной работы: ответ для четырехточечной функции ОМГ с одним вырожденным полем и тремя полями материи в общем положении. Складывая граничные вклады (8.16) и вклад от кривизны (6.5), получим следующее выражение для нормированного корреляционного числа:

$$
Z_{\mathrm{L}}^{-1} \int\left\langle\mathcal{U}_{m, n}(x) \mathcal{W}_{a_{1}} \mathcal{W}_{a_{2}} \mathcal{W}_{a_{3}}\right\rangle d^{2} x=-\left(b^{-2}+1\right) b^{-3}\left(b^{-2}-1\right) \Sigma_{m, n}\left(a_{1}, a_{2}, a_{3}\right)
$$

где

$$
\Sigma_{m, n}\left(a_{1}, a_{2}, a_{3}\right)=-2 m n \lambda_{m, n}+\sum_{i=1}^{3} \sum_{r, s}^{(m, n)}\left(\lambda_{m, n}-\left|a_{i}-\lambda_{r, s}-\frac{Q}{2}\right|_{\operatorname{Re}}\right) .
$$

Это выражение становится несколько более прозрачным после простого пересуммирования членов с $\lambda_{m, n}$ :

$$
\Sigma_{m, n}\left(a_{1}, a_{2}, a_{3}\right)=m n \lambda_{m, n}-\sum_{i=1}^{3} \sum_{r, s}^{(m, n)}\left|\lambda_{i}-\lambda_{r, s}\right|_{\mathrm{Re}}
$$

Здесь для общих вставок полей материи мы ввели удобные “импульсные” параметры $\lambda_{i}=Q / 2-a_{i}$. В этой параметризации симметрия выражения (9.2) относительно замены $a_{i} \rightarrow Q-a_{i}$ (т.е. независимость от выбора “лиувиллевского одевания" полей материи) становится явной, и мы можем предложить следующее диаграммное 
представление:

$$
\left.\left.\Sigma_{m, n}\left(a_{1}, a_{2}, a_{3}\right)=m n \lambda_{m, n}-\sum_{r, s}^{(m, n)}\right\rangle_{3}^{(m, n)}\right\rangle^{2}-
$$

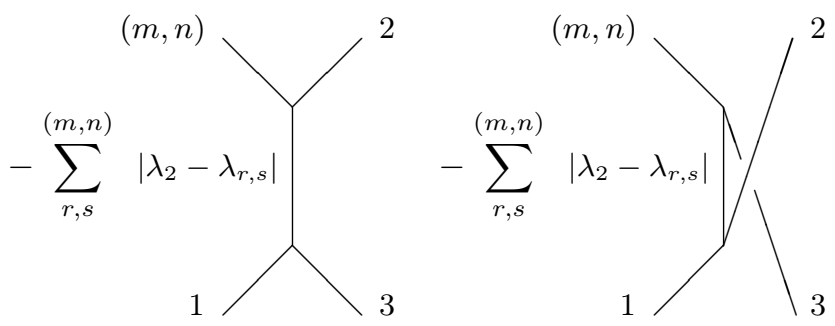

Это представление выглядит исключительно простым, особенно в сравнении с тем трудным путем, которым мы к нему пришли. Вероятнее всего, это означает, что мы до сих пор остаемся в неведении относительно гораздо более простого, а потому и более глубокого взгляда на физику, лежащую в основе данной проблемы. Тем не менее, мы надеемся, что наш ответ верен по крайней мере в случае, когда в нем присутствуют невырожденные поля общего вида вместе с вырожденным составным полем $U_{m, n}$. Некоторые сравнения с результатами прямого численного интегрирования в уравнении (5.1), равно как и с ответами, приходящими из матричных моделей, представлены в следующих двух разделах. Оба эти сравнения подтверждают правильность выражения (9.1).

Однако более глубокое исследование равенства (9.3) вскрывает серьезные проблемы. Существует очевидная несогласованность, если одно (или более) из полей $W_{i}$ в нашей корреляционной функции становится вырожденным примарным полем материи $\Phi_{m^{\prime}, n^{\prime}}$. Эта проблема появляется немедленно, если мы вводим тождественное поле материи $I=\Phi_{1,1}$ (т.е. $W_{3}=W_{b}=V_{b}$ ) вместо одной из общих $W$-вставок. Наша формула в этом случае дает

$$
\Sigma_{m, n}\left(a_{1}, a_{2}, b\right)=m n \lambda_{m, n}-\sum_{r, s}^{(m, n)}\left|\lambda_{1,-1}-\lambda_{r, s}\right|-\sum_{i=1}^{2} \sum_{r, s}^{(m, n)}\left|\lambda_{i}-\lambda_{r, s}\right|_{\mathrm{Re}},
$$

в то время как из стандартной интерпретации поля $V_{b}$ как элемента площади следует (см. в равной степени уравнение (8.8)), что

$$
\Sigma_{m, n}\left(a_{1}, a_{2}, b\right)=b-\sum_{i=1}^{2}\left|\lambda_{i}\right|_{\mathrm{Re}}
$$

и это явно противоречит $(9.5)$, если $(m, n) \neq(1,1)$. Похожая проблема всегда возникает, если вырождение вставки материи $W_{i}$ в одной или более точках приводит 
к уменьшению числа конформных блоков, участвующих в выражении для четырехточечной функции полей материи (2.17). Мы уже отмечали эту трудность как причину того, что мы рассматриваем только случай, когда все три вставки полей $W_{i}$ невырожденны и соответствуют полям в случае общего положения. С аналитической точки зрения этот эффект несомненно обусловлен тонкостями, относящимися к порядку вычисления двух пределов, т.е. $\alpha_{1} \rightarrow \alpha_{m^{\prime}, n^{\prime}}$ в $W_{1}$ и $\lambda_{2} \pm \lambda_{3} \rightarrow \lambda_{r, s}$ (с $\left(r^{\prime}, s^{\prime}\right)$, принадлежащими набору, предписываемому стандартными правилами слияния) в $W_{2}$ и $W_{3}$, что приводит к необходимости взять в качестве $\Phi_{1}$ вырожденное $\left(m^{\prime}, n^{\prime}\right)$ поле ОММ.

В настоящий момент мы не в состоянии разрешить эту существенную трудность. Тем не менее, мы убеждены, что формула (9.2) остается справедливой до тех пор, пока число конформных блоков полей материи остается равным значению уровня вырождения $m n$ для поля $U_{m, n}$. Это поле выбирается в формуле (5.1) как вставка, по которой производится интегрирование. Если это не так, то иногда можно использовать свободу выбора точки интегрирования в формуле (1.17) и взять другое вырожденное поле $U_{m^{\prime}, n^{\prime}}$ в качестве начальной точки, при этом число $m^{\prime} n^{\prime}$ будет давать настоящее число конформных блоков. Например, это всегда можно сделать, если все вырожденные поля принадлежат подмножеству $(1, n)($ или $(m, 1))$. В этом случае достаточно начать с поля с наименьшим значением параметра $n$.

В случае более общих вырожденных вставок это часто не выполняется. Простой пример дается ОР $\Phi_{1,2} \Phi_{2,1}$, которое выражается через всего лишь одно представление $\left[\Phi_{2,2}\right]$, и вся четырехточечная функция с такими двумя вставками операторов материи содержит всего один конформный блок. В связи с указанной трудностью нам представляется полезным рассмотреть пример четырехточечной функции $\left\langle U_{1,2} U_{2,1} U_{a_{1}} U_{a_{2}}\right\rangle_{\text {омг }}$, которая содержит эту пару вставок операторов материи. Разумеется, отщепление сингулярных векторов материи (2.8) требует, чтобы параметры $a_{1}$ и $a_{2}$ были согласованы в соответствии с правилами слияния с "промежуточным" представлением $\left[\Phi_{2,2}\right]$. Рассмотрим случай $a_{1}+a_{2}=Q-\lambda_{1,1}$ и продемонстрируем на его примере интересный и важный эффект. При этом выборе параметров лиувиллевская четырехточечная функция становится резонансной. Это ясно видно при выборе следующих лиувиллевских “одеваний” для полей $\Phi_{1,2}$ и $\Phi_{2,1}$ :

$$
\begin{aligned}
\left\langle U_{1,2} U_{2,1} U_{a} U_{Q / 2-a}\right\rangle_{\mathrm{OM} \Gamma}=\int & \left\langle\Phi_{1,2}(x) \Phi_{2,1}(0) \Phi_{a-b}(1) \Phi_{q / 2-a}(\infty)\right\rangle_{\mathrm{OMM}} \times \\
& \times\left\langle V_{b^{-1}-b / 2}(x) V_{b-b^{-1} / 2}(0) V_{a}(1) V_{Q / 2-a}(\infty)\right\rangle_{\mathrm{L}} d^{2} x,
\end{aligned}
$$

следовательно, $\sum_{i} a_{i}=Q$ в лиувиллевской составляющей. В ЛГ такие полюсы интерпретируются как определенное $(\ln \mu)$-одевание стандартной корреляционной функции с зависимостью от $\mu$, подобной степенной. С другой стороны, каждому читателю, знакомому с техникой матричных моделей (окончательный результат которой весьма похож на картину, возникающую в методе среднего поля), довольно трудно представить, как логарифмическая зависимость может возникнуть в этом контексте. Аналогично наш результат (9.3) при этом никогда не сингулярен относительно параметров $\mu$ и $a_{i}$. Оба подхода предполагают, что даже если лиувиллевская корреляционная функция имеет полюс как функция параметра $a_{i}$, то интеграл по 
пространству модулей дает нуль, который уничтожает эту сингулярность. Это именно тот эффект, который мы желаем продемострировать для случая (9.7), хотя мы еще не в состоянии разрешить эту сингулярность и получить ответ для конечной части интеграла.

Резонансная лиувиллевская корреляционная функция есть

$$
\begin{aligned}
& \left\langle V_{b^{-1}-b / 2}(x) V_{b-b^{-1} / 2}(0) V_{a}(1) V_{Q / 2-a}(\infty)\right\rangle_{\mathrm{L}}= \\
& \quad=-(x \bar{x})^{b^{-2}+b^{2}-5 / 2}[(1-x)(1-\bar{x})]^{a b-2 a b^{-1}} \ln \mu .
\end{aligned}
$$

Четырехточечная функция материи в этом случае тоже имеет довольно простой вид

$$
\left\langle\Phi_{1,2}(x) \Phi_{2,1}(0) \Phi_{a-b}(1) \Phi_{q / 2-a}(\infty)\right\rangle_{\mathrm{OMM}}=\frac{\left(1-b^{2}-(1-2 a b) x\right)\left(1-b^{2}-(1-2 a b) \bar{x}\right)}{\left(1-b^{2}\right)^{2}(x \bar{x})^{1 / 2}[(1-x)(1-\bar{x})]^{b^{2}-a b}} .
$$

Таким образом,

$$
\begin{aligned}
& \left\langle U_{1,2} U_{2,1} U_{a} U_{Q / 2-a}\right\rangle_{\mathrm{OM} \Gamma}= \\
& \quad=-\ln \mu \int \frac{\left(1-b^{2}-(1-2 a b) x\right)\left(1-b^{2}-(1-2 a b) \bar{x}\right)}{\left(1-b^{2}\right)^{2}(x \bar{x})^{3-b^{-2}-b^{2}}[(1-x)(1-\bar{x})]^{b^{2}-2 a b+2 a b^{-1}}} d^{2} x .
\end{aligned}
$$

Интегрирование можно произвести явно с использованием общей формулы ${ }^{9)}$

$$
\int x^{\mu-1} \bar{x}^{\bar{\mu}-1}(1-x)^{\nu-1}(1-\bar{x})^{\bar{\nu}-1} d^{2} x=\frac{\pi \Gamma(\mu) \Gamma(\nu) \Gamma(1-\bar{\mu}-\bar{\nu})}{\Gamma(1-\bar{\mu}) \Gamma(1-\bar{\nu}) \Gamma(\mu+\nu)} .
$$

Это показывает, что интеграл обращается в нуль, что и следовало ожидать на основании общих соображений. Конечный вклад этого интеграла требует более тщательного анализа. Мы надеемся вскоре внести ясность в решение этого важного вопроса.

\section{0. ЧИСЛЕННАЯ ПРОВЕРКА: ПРЯМОЕ ИНТЕГРИРОВАНИЕ}

В этом разделе производится проверка аналитического выражения (9.2) для интеграла (5.1) посредством прямого численного интегрирования по пространству модулей. Разумеется, пространство трех общих параметров $a_{1}, a_{2}$ и $a_{3}$, а также параметра центрального заряда $b$ оказывается слишком обширным для того, чтобы быть исследованным на каком-либо приемлемом уровне понимания. Поэтому ограничимся лишь весьма предварительными рассуждениями, рассмотрев простой пример четы-

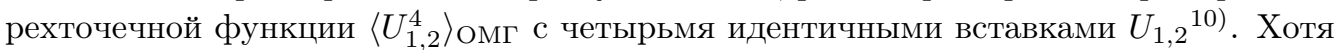
это и не в точности отвечает случаю трех общих невырожденных примарных полей материи, ОР $\Phi_{1,2} \Phi_{1,2}$ всегда содержит два представления $[I]$ и $\left[\Phi_{1,3}\right]$, и потому в корреляционной функции полей материи всегда участвуют два конформных блока. В этом случае вышеприведенный критерий выполняется, следовательно, можно предположить, что ответ формально задается выражениями (9.1) и (9.2).

\footnotetext{
9) В этой формуле предполагается, что $\mu-\bar{\mu}$ и $\nu-\bar{\nu}-$ целые числа, в противном случае интеграл не имеет смысла.

10) Отложим более продвинутый анализ до будущих публикаций.
} 
С другой стороны, этот частный пример обладает преимуществами при численных вычислениях. Структурные константы материи (и до некоторой степени лиувиллевская постоянная) при этом упрощаются. Конформные блоки материи явно выражаются через гипергеометрические функции. И наконец, все четыре вставки идентичны, а потому проинтегрированная 2-форма в выражении (5.1) обладает полной модулярной симметрией, т.е. она инвариантна при преобразованиях

$$
\begin{array}{cc}
R: x \rightarrow 1-x, & T: x \rightarrow 1 / x, \\
R T: x \rightarrow 1 /(1-x), & T R: x \rightarrow 1-1 / x, \\
T R T=R T R: x \rightarrow x /(x-1) .
\end{array}
$$

Это позволяет редуцировать область интегрирования в интеграле (5.1) и рассматривать вместо целой комплексной плоскости фундаментальную область, т.е. сегмент $\mathbf{F}=\{\operatorname{Re} x<1 / 2 ;|1-x|<1\}$.

Общий результат (9.1) для $\left\langle U_{1,2}^{4}\right\rangle$ Омг может быть записан как

$$
Z_{\mathrm{L}}^{-1}\left\langle U_{1,2}^{4}\right\rangle_{\text {OM }}=-(2 \pi)^{4}\left(b^{-2}+1\right) b^{-3}\left(b^{-2}-1\right) \Sigma_{1,2}\left(b^{-2}\right) \mathcal{L}^{4}(g),
$$

где статистическая сумма $Z_{\mathrm{L}}$ та же, что и в $(4.6)$, а “фактор внешней линии” $\mathcal{L}(g)$ имеет вид

$$
\mathcal{L}(g)=\left|\frac{\gamma\left(2 g b-b^{2}\right) \gamma\left(2 g b^{-1}-b^{-2}\right)}{4 \gamma^{2 g / b-1}\left(b^{2}\right) \gamma\left(2-b^{-2}\right)}\right|^{1 / 2} .
$$

Здесь $g$ - решение “условия одевания" (1.14) $)^{11)}$,

$$
g=\frac{Q}{2}-\sqrt{\left(b-\frac{b^{-1}}{2}\right)^{2}}=\frac{b^{-1}}{2}+\frac{b}{2}-\left|b-\frac{b^{-1}}{2}\right| .
$$

Множитель (9.3) при этом приобретает вид

$$
b^{-1} \Sigma_{1,2}\left(b^{-2}\right)=-\frac{1}{2} b^{-2}+\frac{7}{2}-\frac{3}{2}\left|b^{-2}-3\right| .
$$

Этот множитель как функция от $b^{-2}$ представлен сплошной линией на рис. 5 .

Исходный четырехточечный интеграл (5.1) в этом случае превращается в

$$
\left\langle U_{1,2}^{4}\right\rangle_{\mathrm{OM} \Gamma}=6 \int_{\mathbf{F}} G_{\mathrm{M}}(x, \bar{x}) G_{\mathrm{L}}(x, \bar{x}) d^{2} x,
$$

где корреляционная функция $G_{\mathrm{M}}(x, \bar{x})=\left\langle\Phi_{1,2}(x) \Phi_{1,2}(0) \Phi_{1,2}(1) \Phi_{1,2}(\infty)\right\rangle_{\mathrm{OMM}}$ в модели ОММ записывается явно как

$$
\begin{gathered}
G_{\mathrm{M}}(x, \bar{x})=\mathcal{F}_{1,1}(x) \mathcal{F}_{1,1}(\bar{x})-\kappa^{2} \mathcal{F}_{1,3}(x) \mathcal{F}_{1,3}(\bar{x}), \\
\kappa^{2}=\frac{\left(1-2 b^{2}\right)^{2} \gamma\left(b^{2}\right)}{\gamma^{2}\left(2 b^{2}\right) \gamma\left(2-3 b^{2}\right)}
\end{gathered}
$$

\footnotetext{
11) В этом разделе буква $g$ снова имеет значение, отличное от $b^{-2}$.
} 


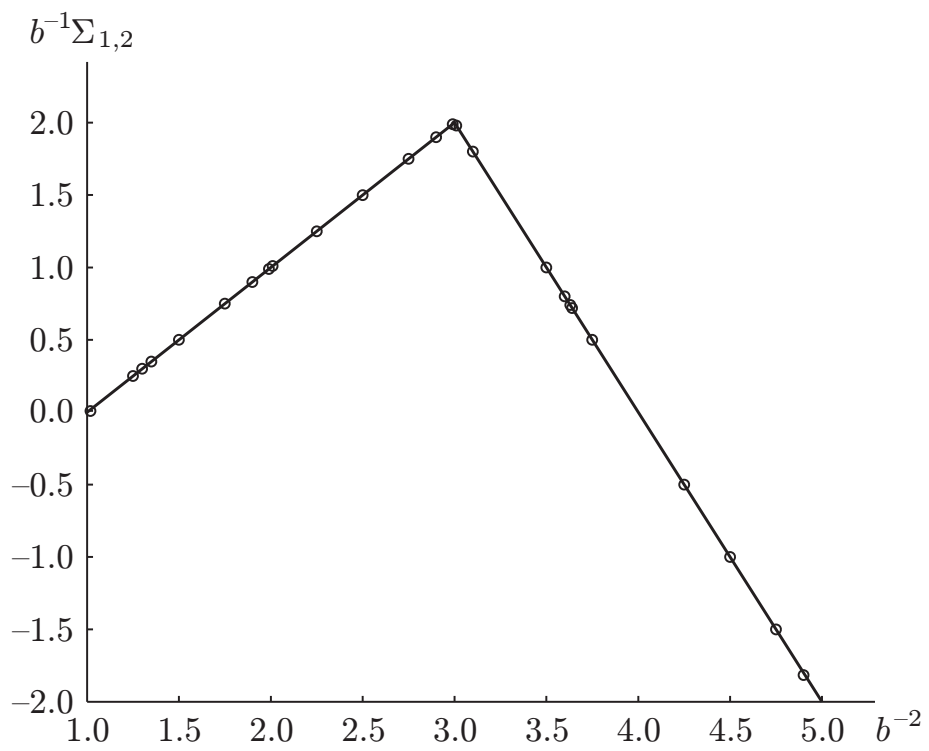

Рис. 5. Прямое численное вычисление интеграла (10.6) (кружки) в сравнении с точным результатом (сплошная ломаная)

и конформные блоки суть

$$
\begin{aligned}
& \mathcal{F}_{1,1}(x)=x^{1-3 b^{2} / 2}(1-x)^{1-3 b^{2} / 2}{ }_{2} F_{1}\left(2-3 b^{2}, 1-b^{2}, 2-2 b^{2}, x\right), \\
& \mathcal{F}_{1,3}(x)=x^{b^{2} / 2}(1-x)^{b^{2} / 2}{ }_{2} F_{1}\left(-1+3 b^{2}, b^{2}, 2 b^{2}, x\right) .
\end{aligned}
$$

Лиувиллевская корреляционная функция $G_{\mathrm{L}}(x, \bar{x})=\left\langle V_{g}(x) V_{g}(0) V_{g}(1) V_{g}(\infty)\right\rangle_{\mathrm{L}}$ может быть представлена в виде

$$
G_{\mathrm{L}}(x, \bar{x})=\mathcal{R}_{g} \int^{\prime} \frac{d P}{4 \pi} r_{g}(P) \mathcal{F}_{P}\left(\begin{array}{cc}
\Delta_{g} & \Delta_{g} \\
\Delta_{g} & \Delta_{g}
\end{array} \mid x\right) \mathcal{F}_{P}\left(\begin{array}{cc}
\Delta_{g} & \Delta_{g} \\
\Delta_{g} & \Delta_{g}
\end{array} \mid \bar{x}\right)
$$

где сложные выражения для лиувиллевских структурных констант сводятся к равенствам

$$
\begin{gathered}
\mathcal{R}_{g}=\left(\gamma\left(b^{2}\right) b^{2-2 b^{2}}\right)^{(Q-4 g) / b} \frac{\Upsilon_{b}^{4}(b) \Upsilon_{b}^{4}(2 g)}{\pi^{2} \Upsilon_{b}^{4}(2 g-Q / 2)} \\
r_{g}(P)=\frac{\pi^{2} \Upsilon_{b}(2 i P) \Upsilon_{b}(-2 i P) \Upsilon_{b}^{4}(2 g-Q / 2)}{\Upsilon_{b}^{2}(b) \Upsilon_{b}^{2}(2 g-Q / 2-i P) \Upsilon_{b}^{2}(2 g-Q / 2+i P) \Upsilon_{b}^{4}(Q / 2-i P)}= \\
=\operatorname{sh} 2 \pi b^{-1} P \operatorname{sh} 2 \pi b P \exp \left(-8 \int_{0}^{\infty} \frac{d t}{t} \frac{\sin ^{2} P t\left(\operatorname{ch}^{2}(Q-2 g) t-e^{-Q t} \cos ^{2} P t\right)}{\operatorname{sh} b t \operatorname{sh} b^{-1} t}\right) .
\end{gathered}
$$

Симметричный конформный блок общего вида $\mathcal{F}_{P}\left(\begin{array}{cc}\Delta_{g} & \Delta_{g} \\ \Delta_{g} & \Delta_{g}\end{array} \mid x\right)$ может быть найден численно с помощью рекурсивной процедуры, введенной в [19] (обзор этого подхода 
можно найти в более доступной работе [3]). Как обычно, штрих при знаке интеграла обозначает возможные дискретные члены. В этом исследовании рассматривается только область $1<b^{-2}<5$, в которой такие лишние члены не возникают, и интеграл в выражении (10.10) имеет обычный смысл. На рис. 5 результаты численного вычисления интеграла (10.6) показаны кружками. Заметим, что вблизи излома в точке $b^{-2}=3$ этот интеграл в окрестностях точек $x=0,1$ и $\infty$ сходится очень медленно. Числа на рис. 5 в этой области можно получить только после некоторой модификации алгоритма интегрирования. Решение этой проблемы вместе с другими интересными деталями, связанными с численным расчетом интеграла (10.6), будут предметом отдельной публикации.

\section{1. СРАВНЕНИЕ С МАТРИЧНЫМИ МОДЕЛЯМИ}

Разумеется, представляет значительный интерес сравнение результата (9.1) с корреляционными числами, возникающими в контексте матричных моделей. $\mathrm{K}$ сожалению, не существует прямого способа провести это сравнение. Стандартная формулировка матричных моделей в основном интерпретируется в терминах собственно рациональных минимальных моделей с $b^{2}=p / p^{\prime}$ и включает в себя только вырожденные поля материи КТП. Более того, основной массив матричномодельных результатов содержит теоретико-полевую информацию в достаточно зашифрованном виде. Требуются значительные усилия по извлечению соответствующих корреляционных функций и по их интерпретированию в терминах МГ.

Имеется пример матричной модели, в которой непрерывная интерпретация однозначна, и к тому же центральный заряд полей материи изменяется непрерывным образом, так что наш подход ОМГ представляется вполне подходящим. Недавно Костов [20] получил новый интересный результат в так называемой гравитационной $O(n)$-модели, представляющей собой модель на случайной решетке, покрытой полимерными петлями без пересечений, каждая компонента которой несет весовой фактор $n$. Критическая термодинамика контролируется двумя параметрами: “космологической постоянной" $x$, связанной с размером решетки, и "массовым" параметром $t$, регулирующим длину полимеров. Оба эти параметра выбираются как отклонения от параметров в двойной критической точке, в которой размер решетки и длина петель "раздуваются" одновременно. Критическая сингулярность в окрестности этой точки становится предметом рассмотрения в непрерывной теории поля.

В соответствии с результатами работы [20] сингулярная часть $Z(t, x)$ статистической суммы для рода ноль допускает следующее простое описание. Введем (стандартную для $O(n)$-модели) параметризацию веса петли $-2<n<2$,

$$
n=-2 \cos (\pi g),
$$

в терминах переменной $1<g<2$. Пусть также $Z(x, t)$ обозначает сингулярную часть статистической суммы для рода ноль, а

$$
u=-(g-1) Z_{x x}
$$

- ее вторую производную по $x$. Тогда $u$ - решение следующего простого трансцендентного уравнения:

$$
u^{p}+t u^{p-1}=x
$$


где $p=(g-1)^{-1}$. Уравнения (11.2) и (11.3) приводят к следующему разложению:

$$
\begin{aligned}
Z & =t x^{2}+x^{g+1} \sum_{\substack{n=0 \\
n \neq 1}}^{\infty} \frac{\Gamma(g(n-1)-n-1)}{n ! \Gamma(g(n-1)-2 n+2)}\left(t x^{1-g}\right)^{n}= \\
& =x^{g+1}\left(\frac{-1}{(g-1) g(g+1)}+t x^{1-g}+\frac{\left(t x^{1-g}\right)^{2}}{2(g-3)}+\frac{\left(t x^{1-g}\right)^{3}}{6}+\frac{(g-2)\left(t x^{1-g}\right)^{4}}{8}+\cdots\right) .
\end{aligned}
$$

С другой стороны, критические разреженные полимеры на этой случайной решетке допускают стандартную непрерывную параметризацию в терминах ОМГ с

$$
c_{\mathrm{M}}=13-6\left(g+g^{-1}\right)
$$

(см. работу [21]). Это один из примеров, когда ОММ $\mathcal{M}_{b^{2}}$ проявляет себя как теория полей материи, параметры которой связаны соотношением

$$
g=b^{-2} \text {. }
$$

Более того, оператор ОММ, связанный с внекритической "массой” полимерной петли, скорее всего, представляет собой вырожденное поле $\Phi_{1,3}$, и мы имеем дело с ОМГ, возмущенной композитным полем

$$
U_{1,3}=\Phi_{1,3} V_{b^{-1}-b}
$$

Отметим частный выбор лиувиллевского “одевания", который имеет место в контексте гравитации при $1<b^{-2}<3$ [21], [22]. Таким образом, коэффициенты в разложении (11.4) можно интерпретировать как многоточечные корреляционные функции этого поля в соответствующей теории ОМГ. Так как общая нормировка статистической суммы зависит от масштаба и не может быть фиксирована универсальным способом, естественно связать нормированные корреляционные функции соотношением

$$
Z_{\mathrm{L}}^{-1}\left\langle U_{1,3}^{n}\right\rangle_{\mathrm{OM} \Gamma}=\frac{\Gamma\left(b^{-2}(n-1)-n-1\right) \Gamma\left(2-b^{-2}\right)}{\Gamma\left(b^{-2}(n-1)-2 n+2\right) \Gamma\left(-1-b^{-2}\right)}\left(2 \pi L_{\mathrm{eg}}\right)^{n} .
$$

Здесь введен масштабный множитель $L_{\mathrm{eg}}$, поскольку нормировка размерной космологической постоянной $\mu$ отличается от нормировки $x$, а также поле $U_{1,3}^{(\mathrm{mat})}$, соответствующее параметру $t$, нормируется способом, отличным от нормировки $U_{1,3}$. Масштабный множитель связывает между собой безразмерные комбинации:

$$
U_{1,3}^{(\mathrm{mat})} x^{g-1}=\left(2 \pi L_{\mathrm{eg}}\right)^{-1} U_{1,3}
$$

Этот множитель легко найти явно, сравнивая двух- и трехточечные функции ОМГ (4.9) с соответствующими членами в (11.8) [7]:

$$
L_{\mathrm{eg}}=\frac{\gamma\left(b^{-2}-1\right)\left[-\gamma\left(b^{2}\right) \gamma\left(2-3 b^{2}\right)\right]^{1 / 2}}{2\left(2-b^{-2}\right)\left(\pi \mu \gamma\left(b^{2}\right)^{b^{-2}-1}\right)} .
$$

2 Теоретическая и математическая физика, т. 147, № 3, 2006 г. 
Четырехточечный коррелятор (11.8) имеет вид

$$
Z_{\mathrm{L}}^{-1}\left\langle U_{1,3}^{4}\right\rangle_{\mathrm{OM} \Gamma}=-3(g-2)(g-1) g(g+1)\left(2 \pi L_{\mathrm{eg}}\right)^{4} .
$$

В то же время, выражение (9.1) в этом случае дает

$$
\left\langle\left\langle\mathcal{U}_{1,3}^{4}\right\rangle\right\rangle=-(g+1) g(g-1) b^{-1} \Sigma_{1,3},
$$

где

$$
b^{-1} \Sigma_{1,3}=\frac{3}{2}(g+3-|g-1|-|g-3|-|g-5|),
$$

и при $1<g<3$ имеем

$$
b^{-1} \Sigma_{1,3}=3(g-2),
$$

что находится в согласии с (11.11).

Благодарности. Авторы признательны Б. Фейгину, И. Костову, А. Литвинову, В. Петковой и А. Замолодчикову за полезные обсуждения. Наше сотрудничество было поддержано INTAS (грант INTAS-OPEN-03-51-3350). Работа А. Белавина была поддержана РФФИ (грант № 04-02-16027), программой РАН “Элементарные частицы и фундаментальная ядерная физика", Программой поддержки научных школ (грант № 2044.2003.2), а также Фондом Александра Гумбольдта. Как обычно, Ал. Замолодчиков был во многом воодушевлен Галиной Гриценко. Кроме того, Ал. Замолодчиков благодарит за гостеприимство и стимулирующую научную атмосферу Лабораторию теоретической физики RIKEN, где была написана основная часть данной работы. Его работа была также поддержана Европейской Комиссией по контракту EUCLID HRPN-CT-2002-00325. Организаторы конференции "RAQIS 05” в LAPP обеспечили авторам редкую возможность работать вдоволь в личном контакте и в результате согласовать окончательную версию статьи.

\section{Список литературы}

[1] A. Polyakov, Phys. Lett. B, 103 (1981), 207.

[2] H. Dorn, H.-J. Otto, Phys. Lett. B, 291 (1992), 39; hep-th/9206053; Nucl. Phys. B, 429 (1994), 375; hep-th/9403141; A. Zamolodchikov, Al. Zamolodchikov, Nucl. Phys. B, 477 (1996), 577.

[3] J. Teschner, Class. Quant. Grav., 18 (2001), R153; hep-th/0104158.

[4] A. Belavin, A. Polyakov, A. Zamolodchikov, Nucl. Phys. B, 241 (1984), 333.

[5] Al. Zamolodchikov, Int. J. Mod. Phys. A, 19S2 (2004), 510; hep-th/0312279.

[6] V. Dotsenko, V. Fateev, Phys. Lett. B, 154 (1985), 291.

[7] Ал. Б. Замолодчиков, ТМФ, 142:2 (2005), 218; hep-th/0505063.

[8] H. Poincaré, J. Math. Pure Appl., 5:4 (1898), 137; А. М Поляков, Неопубликованные лекиии, 1979.

[9] L. Benoit, Y. Saint-Aubin, Phys. Lett. B, 215 (1988), 517.

[10] C. Imbimbo, S. Mahapatra, S. Mukhi, Nucl. Phys. B, 375 (1992), 399. 
[11] B. Feigin, Private communication, 2005.

[12] I. Klebanov, A. Polyakov, Mod. Phys. Lett. A, 6 (1991), 3273; hep-th/9109032.

[13] E. Witten, Nucl. Phys. B, 373 (1992), 187; hep-th/9108004.

[14] И. К. Костов, В. Б. Петкова, ТМФ, 146:1 (2006), 132; hep-th/0505078.

[15] N. Seiberg, D. Shih, JHEP, 0402 (2004), 021; hep-th/0312170.; M. Douglas, I. Klebanov, D. Kutasov, J. Maldacena, E. Martinec, N. Seiberg, A new hat for the $c=1$ matrix model, hep-th/0307195.

[16] A. A. Belavin, Al. B. Zamolodchikov, Писъма в ЖЭТФ, 82 (2005), 8.

[17] D. Kutasov, E. Martinec, N. Seiberg, Phys. Lett. B, 276 (1992), 437; hep-th/9111048.

[18] N. Seiberg, "Notes on quantum Liouville theory and quantum gravity", Random Surfaces and Quantum Gravity, Proc. NATO Adv. Res. Workshop (Cargese, France, May 27 June 2, 1990), NATO ASI Ser., Ser. B, V. 262, eds. O. Alvarez, E. Marinari, P. Windey, Plenum, NY, 1990, 363.

[19] Al. Zamolodchikov, Commun. Math. Phys., 96 (1984), 419; Ал. Б. Замолодчиков, ТМФ, 73:1 (1987), 103.

[20] I. K. Kostov, Termal flow in the gravitational $O(n)$ model, hep-th/0602075.

[21] I. K. Kostov, Mod. Phys. Lett. A, 4 (1989), 217.

[22] M. Gaudin, I. Kostov, Phys. Lett. B, 220 (1989), 200.

Поступила в редакцию 27.Х.2005 г. 